\title{
Empty Convex Hexagons in Planar Point Sets
}

\author{
Tobias Gerken
}

Received: 13 July 2005 / Revised: 4 September 2006 /

Published online: 11 September 2007

(C) Springer Science+Business Media, LLC 2007

\begin{abstract}
Erdős asked whether every sufficiently large set of points in general position in the plane contains six points that form a convex hexagon without any points from the set in its interior. Such a configuration is called an empty convex hexagon. In this paper, we answer the question in the affirmative. We show that every set that contains the vertex set of a convex 9-gon also contains an empty convex hexagon.
\end{abstract}

Keywords Erdős-Szekeres problem · Ramsey theory · Convex polygons and polyhedra $\cdot$ Empty hexagon problem

\section{Introduction}

In 1935, Erdős and Szekeres [5] proved that for each positive integer $n$ there exists a smallest positive integer $g(n)$ such that every planar set of at least $g(n)$ points in general position contains $n$ points that are the vertices of a convex $n$-gon. Here, general position means that no three points are collinear.

The best known bounds for $g(n)$ are $2^{n-2}+1 \leq g(n) \leq\left(\begin{array}{c}2 n-5 \\ n-2\end{array}\right)+1$. The lower bound is due to Erdôs and Szekeres [6] and the upper bound was established recently by Tóth and Valtr [11]. The lower bound is sharp for $n \leq 5$ and is conjectured to be sharp for all $n$ by Erdős and Szekeres [5, 6]. For a survey of results related to the Erdős-Szekeres theorem, see [1, 2, 9, 11].

In 1978, Erdős [3, 4] posed the problem of determining the smallest positive integer $h(n)$, if it exists, such that any set $X$ of at least $h(n)$ points in general position in the plane contains $n$ points that are the vertices of an empty convex polygon; that is, a convex $n$-gon whose interior does not contain any point of $X$. Trivially, $h(n)=n$

\section{T. Gerken (凶)}

Zentrum Mathematik, Technische Universität München, Boltzmannstr. 3,

85747 Garching, Germany

e-mail: gerken@ma.tum.de 
for $n \leq 3$. It is easy to see that $h(4)=5$. In 1978, Harborth [7] proved that $h(5)=10$, while Horton [8] showed in 1983 that for all $n \geq 7, h(n)$ does not exist. The problem of determining the existence of $h(6)$ has since been open. Based on computer experiments, Overmars [10] showed that $h(6) \geq 30$ (if it exists). In this paper, we prove the following theorem which implies that every sufficiently large planar point set in general position contains the vertex set of an empty convex hexagon.

Theorem $1 h(6) \leq g(9)$.

The above bounds yield $129 \leq g(9) \leq 1717$. Note that there exist sets of points without empty convex hexagons that have eight points on the convex hull [10].

\section{Overview of the Proof}

Proof In the following, let $X$ be a finite planar set of points in general position that contains the vertex set of a convex 9-gon. By the Erdős-Szekeres theorem [5] this is always the case if $|X| \geq g(9)$. Let $H \subseteq X$ be the vertex set of a convex 9-gon in $X$ with the minimum $|X \cap \operatorname{conv}(H)|$, where $\operatorname{conv}(M)$ denotes the convex hull of the set $M$. Let $I:=\operatorname{conv}(H) \cap(X \backslash H)$ be the set of points of $X$ inside the convex hull of $H$. Note that $\operatorname{conv}(I)$ is a convex polygon and denote by $\partial I$ its vertex set. If $|I|>2$, let $J:=\operatorname{conv}(I) \cap(X \backslash \partial I)$ be the set of points of $X$ inside the convex hull of $\partial I$. Note that $\operatorname{conv}(J)$ is again a convex polygon and denote by $\partial J$ its vertex set; see Fig. 1. Let $i:=|\partial I|$ and $j:=|\partial J|$. Note that $0 \leq i, j \leq 8$ as otherwise there would be a 9-gon $H^{\prime}$ with smaller $\left|X \cap \operatorname{conv}\left(H^{\prime}\right)\right|$. This leaves the 57 cases $0 \leq i \leq 2$ and $(i, j) \in\{3, \ldots, 8\} \times\{0, \ldots, 8\}$. We argue that in each case either an empty convex $u$-gon can be found $(u \geq 6)$ or a convex 9-gon $H^{\prime}$ with smaller $\left|X \cap \operatorname{conv}\left(H^{\prime}\right)\right|$ is present which contradicts the minimality condition imposed on $H$. (More precisely, the vertex set of an empty convex $u$-gon can be found. In the following, we do not make this distinction when the meaning is clear from the context.)

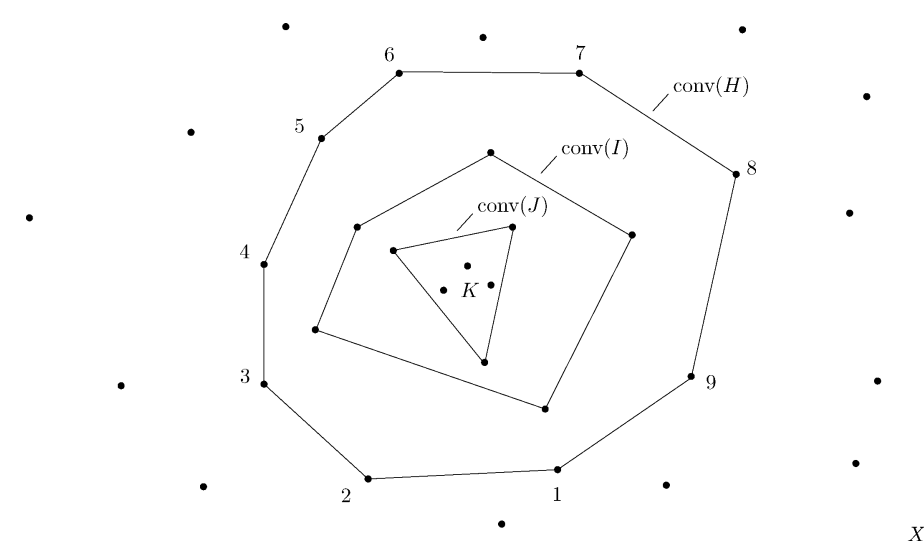

Fig. 1 Basic notation 
Table 1 Overview of the proof structure: for example, the proof for the case $(8,5)$ is given in Sects. 6 (special case) and 10 (general case)

\begin{tabular}{llllllllll}
\hline$i / j$ & 0 & 1 & 2 & 3 & 4 & 5 & 6 & 7 & 8 \\
\hline 0 & 3 & - & - & - & - & - & - & - & - \\
1 & 3 & - & - & - & - & - & - & - & - \\
2 & 3 & - & - & - & - & - & - & - & - \\
3 & 4 & 4 & 4 & 4 & 4 & 4 & 4 & 4 & 4 \\
4 & 5 & 5 & 5 & 5 & 5 & 5 & 5 & 5 & 5 \\
5 & 6 & 7.1 & 8 & 8 & 8 & 8 & 8 & 8 & 8 \\
6 & 3 & 7.2 & 7.3 & $4 / 5$ & 9 & 9 & 9 & 9 & 9 \\
7 & 3 & 7.3 & 3 & $4 / 5$ & 5 & $6 / 10$ & $3 / 10$ & $3 / 10$ & $3 / 10$ \\
8 & 3 & 3 & 3 & 4 & 5 & $6 / 10$ & $3 / 10$ & $3 / 10$ & $3 / 10$ \\
\hline
\end{tabular}

\subsection{Notation}

We use $(i, j)$ to denote a specific case, where $i$ and $j$ are defined as above. Sometimes we use the notation $(i, j, k)$, where $k$ refers to the number of points of $X$ inside the convex hull of $J$; that is, the cardinality of $K:=\operatorname{conv}(J) \cap(X \backslash \partial J)$. The notation $\geq x$ indicates that $x$ is a lower bound for $i, j$ or $k$. Refer to Table 1 for locating the proof of a specific case.

\subsection{Definitions}

Given three points in general position, $P, Q, R$, define the halfplane $H_{P Q}(R)$ as the open halfplane defined by the line $\overline{P Q}$ that contains $R$. A convex chain is a set of consecutive vertices of a convex polygon. Given a convex chain of three points, $\overline{A B C}$, the 3 -sector specified by this chain is defined as

$$
(A B C):=\left[H_{A B}(C) \cap H_{B C}(A)\right] \backslash \operatorname{conv}(\{A, B, C\}) .
$$

Note that three points in general position, $S, T, U$, lying in $(A B C)$ can be used to construct a convex hexagon if $A, B, C \in(S T U)$; see Fig. 2a.

Given a convex chain of four points, $\overline{A B C D}$, the corresponding 4-sector is defined as

$$
(A B C D):=[(A B C) \cap(B C D)] \backslash \operatorname{conv}(\{A, B, C, D\}) .
$$

Note that two points, $S, T$, lying in $(A B C D)$ can be used to construct a convex hexagon if the line $\overline{S T}$ does not intersect conv $(\{A, B, C, D\})$; see Fig. 2b. This means that by construction, given an edge $P Q$ of $\operatorname{conv}(I)$ (respectively conv $(J)$ ), at most three vertices of $\operatorname{conv}(H)$ (respectively $\operatorname{conv}(I))$ can lie in an open halfplane that is defined by the line $\overline{P Q}$ and does not include any other point of $I$ (respectively $J$ ) if no empty convex hexagon is to occur. In the following figures, we use the notation $(P Q)$ to hint to this fact; see Fig. 2c.

Finally, given a convex chain of five points, $\overline{A B C D E}$, the corresponding 5-sector is defined as

$$
(A B C D E):=[(A B C D) \cap(B C D E)] \backslash \operatorname{conv}(\{A, B, C, D, E\}) .
$$




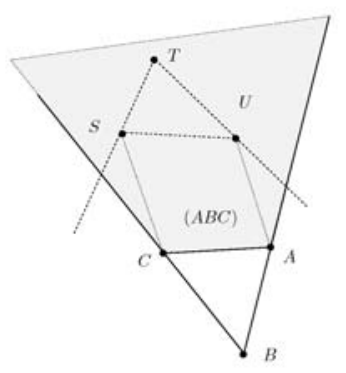

(a)

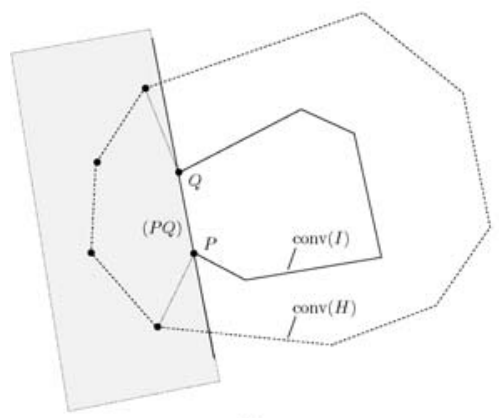

(c)

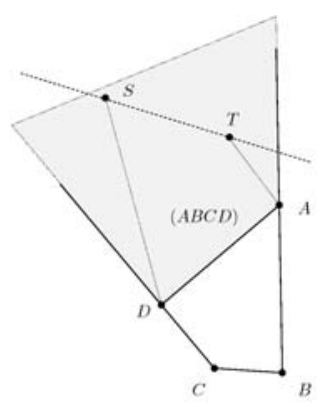

(b)

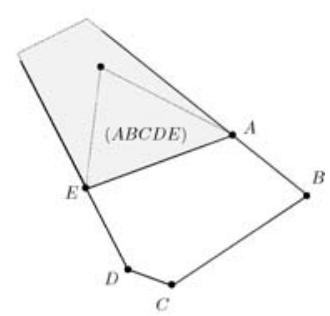

(d)

Fig. 2 Definition: sector

Note that a single point lying in $(A B C D E)$ can be used to construct a convex hexagon; see Fig. 2d.

\section{Elementary Cases}

Note that the cases $(\mathbf{0}, \mathbf{0}),(\geq \mathbf{6 , 0})$ and $(\geq \mathbf{3}, \geq \mathbf{6 , 0})$ are trivial as an empty convex hexagon is present. The cases $(\mathbf{1}, \mathbf{0})$ and $(\mathbf{8}, \mathbf{1})$ can be dealt with by considering a line through the single interior point and one of the vertices of the convex 9- respectively 8 -gon. Due to the general position, on one side of this line a convex chain of four vertices must be present which together with the two preselected points can be used to construct an empty convex hexagon. A similar argument settles the cases $(\mathbf{2}, \mathbf{0})$, $(8,2)$ and $(7,2)$.

\section{The Cases $(3, \geq 0)$ and $(\geq 6,3)$}

We approach the cases $(3, \geq 0)$ and $(\geq 6,3)$ in two batches:

\subsection{The Cases $(3, \geq 0)$ and $(8,3)$}

Follow the notation as indicated in Fig. 3. The variables stand for the number of vertices of the convex 9- respectively 8 -gon in each sector. Assume that no empty 
Fig. 3 Notation for the cases $(3, \geq 0)$ and $(\geq 6,3)$

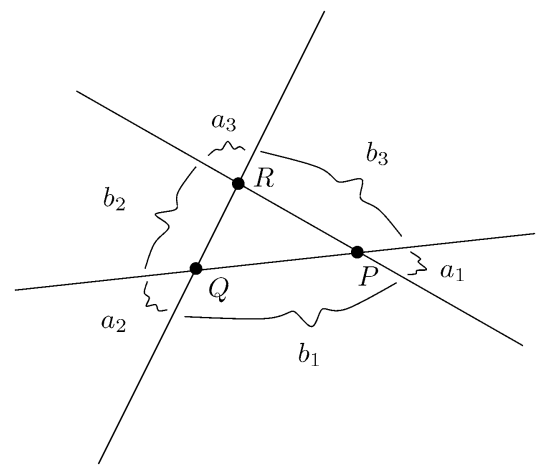

convex hexagon is present. Note that

$$
\begin{aligned}
& 1 \leq a_{1}+b_{1}+a_{2} \leq 3, \\
& 1 \leq a_{2}+b_{2}+a_{3} \leq 3, \\
& 1 \leq a_{3}+b_{3}+a_{1} \leq 3
\end{aligned}
$$

by construction and as otherwise a convex chain of four vertices together with two vertices of the triangle could be used to form an empty convex hexagon. Also,

$$
0 \leq b_{i} \leq 2 \quad(1 \leq i \leq 3)
$$

as otherwise a convex chain of three points together with two vertices of the triangle and either the third vertex of the triangle or (if existent) one of its interior points can be used to form an empty convex hexagon. Summing up the upper bounds in (4.1-4.4) yields

$$
2 \cdot \sum_{i=1}^{3}\left(a_{i}+b_{i}\right) \leq 15 .
$$

Therefore, at most seven vertices can be placed around the triangle and in the two cases at hand an empty convex hexagon is present.

\subsection{The Cases $(6,3)$ and $(7,3)$}

The cases $(6,3,0)$ and $(7,3,0)$ can be settled by a careful investigation of the $\left(a_{1}, b_{1}, a_{2}, b_{2}, a_{3}, b_{3}\right)$-tuples that are feasible for the set of constraints (4.1-4.4). Note that tuples $\left(a_{i}, b_{i}, a_{i+1}\right)$ with $a_{i}=a_{i+1}=0$ are not feasible, as a convex 9-gon $H^{\prime}$ with smaller $\left|X \cap \operatorname{conv}\left(H^{\prime}\right)\right|$ could be constructed; see Fig. 4. In Fig. 4a, replace the vertices of the 9-gon lying in the union of sectors $(A Q B)$ and $(B R C)$ (at least one by construction and at most four in total if no empty convex hexagon is present) by points from the convex chain $\overline{A Q R C}$ of length four. In Fig. 4b, accordingly replace the at most four vertices of the 9-gon lying in the union of sectors $\left(A Q B_{1}\right)$, $\left(B_{1} Q P R B_{2}\right)$ and $\left(B_{2} R C\right)$. 


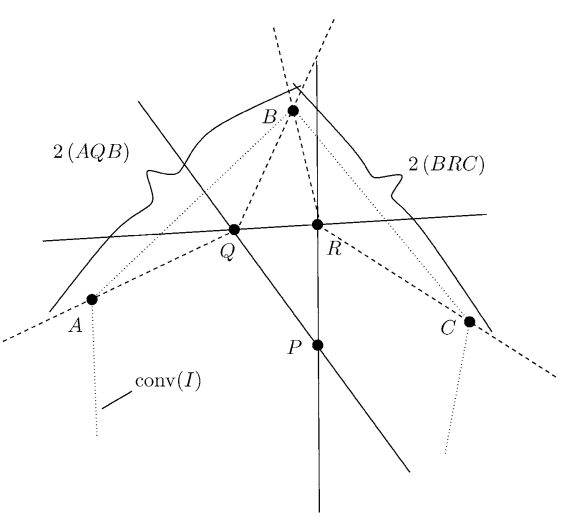

(a)

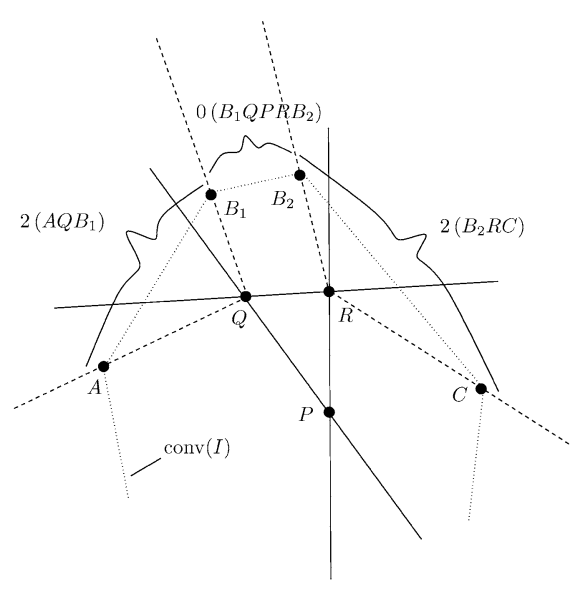

(b)

Fig. $4(x, 3)$ : degenerate cases with $\left(a_{i}, b_{i}, a_{i+1}\right)=(0,1,0)$ and $\left(a_{i}, b_{i}, a_{i+1}\right)=(0,2,0)$ respectively. Numbers indicate the number of vertices of the 9-gon that can lie in each sector without forming an empty convex hexagon

Table 2 Cases $(6,3,0)$ and $(7,3,0)$ : Combinatorial subcases under the assumptions (i) $a_{1} \geq a_{2} \geq a_{3}$ and (ii) $b_{2} \geq b_{3}$ for fixed $\left(a_{1}, b_{1}, a_{2}\right)$

\begin{tabular}{|c|c|c|c|c|c|c|}
\hline$a_{1}$ & $a_{2}$ & $a_{3}$ & $b_{1}$ & $b_{2}$ & $b_{3}$ & Solution \\
\hline 3 & 0 & 0 & $*$ & $*$ & $*$ & Infeasible \\
\hline 2 & 1 & 1 & 0 & $(\leq 1)$ & 0 & $\sum_{i=1}^{3}\left(a_{i}+b_{i}\right) \leq 5$ \\
\hline 2 & 1 & 0 & 0 & $(\leq 2)$ & $(\leq 1)$ & $(2,0,1,2,0,1)$ \\
\hline 2 & 0 & 0 & $*$ & $*$ & $*$ & Infeasible \\
\hline 1 & 1 & 1 & $(\leq 1)$ & $(\leq 1)$ & $(\leq 1)$ & $(1,1,1,1,1,1)$ \\
\hline 1 & 1 & 0 & 1 & 2 & 2 & $(1,1,1,2,0,2)$ \\
\hline 1 & 1 & 0 & 1 & 2 & 1 & $(1,1,1,2,0,1)$ \\
\hline 1 & 1 & 0 & 1 & 2 & 0 & $\sum_{i=1}^{3}\left(a_{i}+b_{i}\right)=5$ \\
\hline 1 & 1 & 0 & 1 & $(\leq 1)$ & $(\leq 1)$ & $\sum_{i=1}^{3}\left(a_{i}+b_{i}\right) \leq 5$ \\
\hline 1 & 1 & 0 & 0 & 2 & 2 & $(1,0,1,2,0,2)$ \\
\hline 1 & 1 & 0 & 0 & $(\leq 2)$ & $(\leq 1)$ & $\sum_{i=1}^{3}\left(a_{i}+b_{i}\right) \leq 5$ \\
\hline 1 & 0 & 0 & $*$ & $*$ & $*$ & Infeasible \\
\hline 0 & 0 & 0 & $*$ & $*$ & $*$ & Infeasible \\
\hline
\end{tabular}

* Marks an arbitrary entry

Note that constraint (4.1) implies $a_{1}+a_{2} \leq 3$

Now assume without loss of generality that (i) $a_{1} \geq a_{2} \geq a_{3}$ and (ii) $b_{2} \geq b_{3}$ for fixed $\left(a_{1}, b_{1}, a_{2}\right)$; see Fig. 3 . Then the only solutions to the above set of constraints (modulo rotations and reflections) are $(2,0,1,2,0,1),(1,1,1,1,1,1)$, 


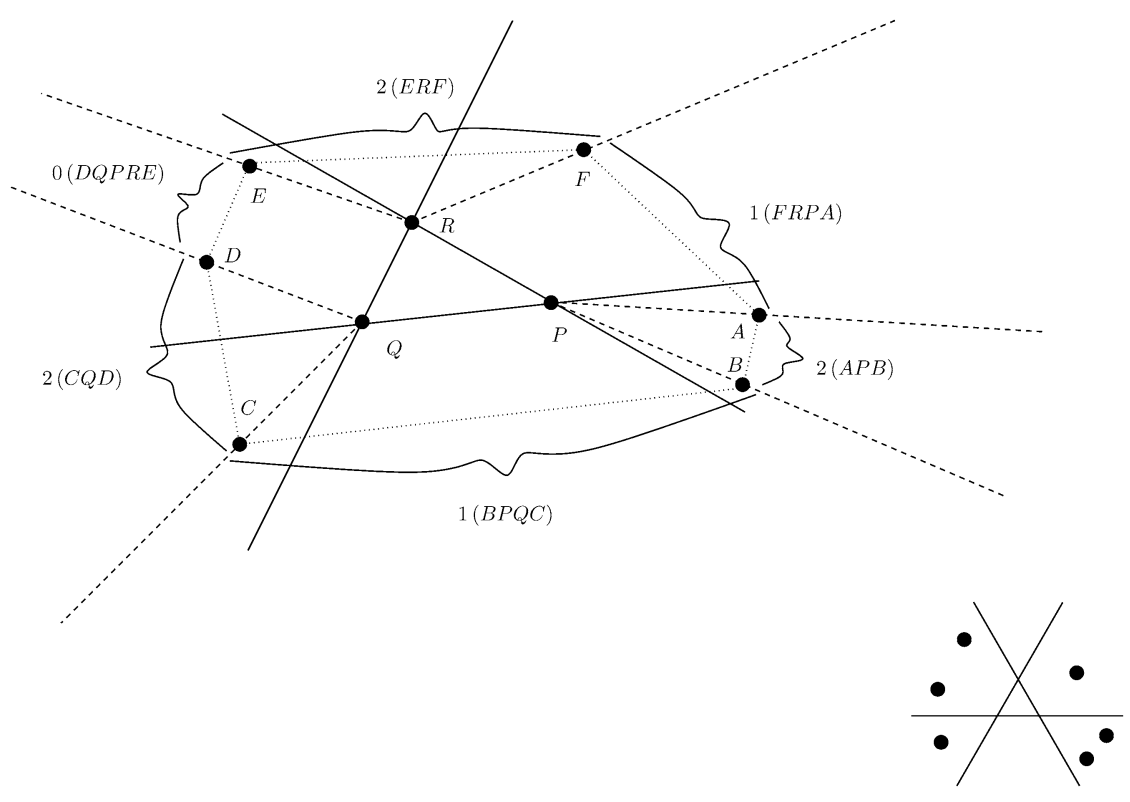

Fig. 5 The case $(6,3,0)$ with $(2,0,1,2,0,1)$

$(1,1,1,2,0,2),(1,1,1,2,0,1)$ and $(1,0,1,2,0,2)$; see Table 2 . These can be treated individually as follows:

- The subcase $(2,0,1,2,0,1)$ can be treated as indicated in Fig. 5. Here and in the following, numbers indicate the number of vertices of the outer polygon that can lie in each sector without forming an empty convex hexagon. As the union of sectors allows for at most eight points in convex position in the outmost layer, due to the presence of a convex 9-gon an empty convex hexagon must occur.

- Figure 6 indicates how to settle the subcase $(1,1,1,1,1,1)$, provided the vertex $Q$ of triangle $P Q R$ lies inside the triangle $B D F$. In that case the quadrilateral $B Q D C$ exists. Similarly we can treat the case that some other of the points $P, Q$ lies inside the triangle $B D F$. If none of the points $P, Q, R$ lies inside the triangle $B D F$, the empty convex hexagon $P B Q D R F$ occurs.

- Figure 7 indicates how to settle the subcase $(1,1,1,2,0,2)$, provided that the point $Q$ lies outside the triangle $B C D$. In that case the quadrilateral $C B Q D$ exists. If $Q$ lies inside the triangle $B C D$, the empty convex hexagon $B Q D E R P$ occurs.

- The subcase $(1,1,1,2,0,1)$ can be treated as indicated in Fig. 8.

- Figure 9 indicates how to settle the subcase $(1,0,1,2,0,2)$.

The proof for the cases $(\mathbf{6}, \mathbf{3}, \geq \mathbf{1})$ and $(\mathbf{7}, \mathbf{3}, \mathbf{\geq} \mathbf{1})$ is given in the following Sect. 5 .

\section{The Cases $(4, \geq 0)$ and $(\geq 7,4)$}

The cases $(\mathbf{4}, \geq 0)$ and $(\geq \mathbf{7}, \mathbf{4})$ can be dealt with simultaneously in three steps: 


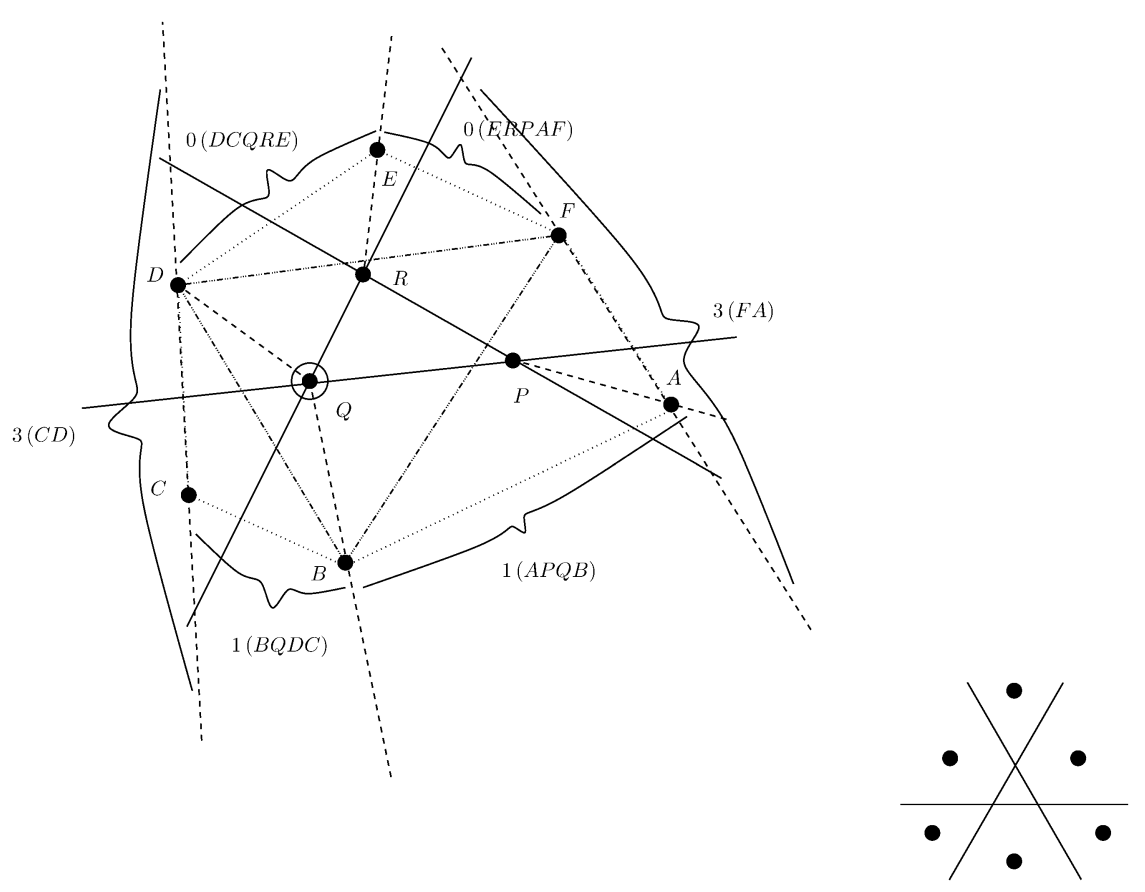

Fig. 6 The case $(6,3,0)$ with $(1,1,1,1,1,1)$. It is assumed that $Q \in \triangle B D F$

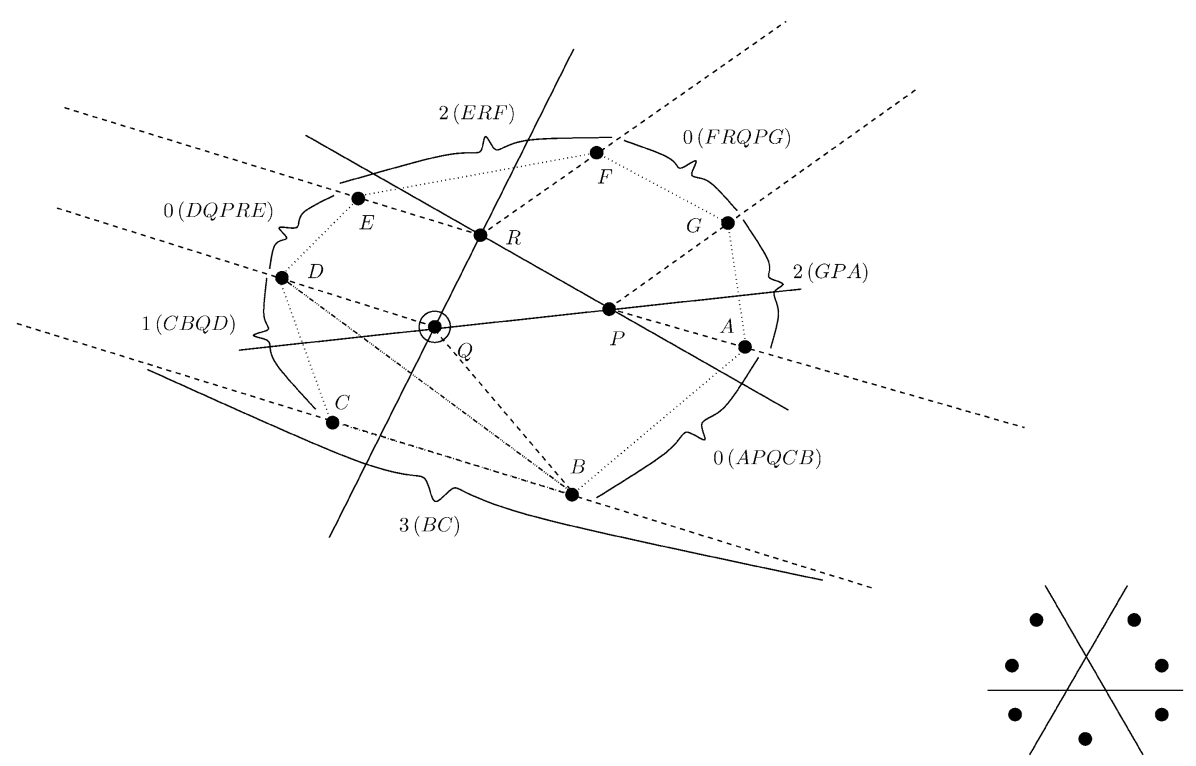

Fig. 7 The case $(7,3,0)$ with $(1,1,1,2,0,2)$. It is assumed that $Q \notin \triangle B C D$ 


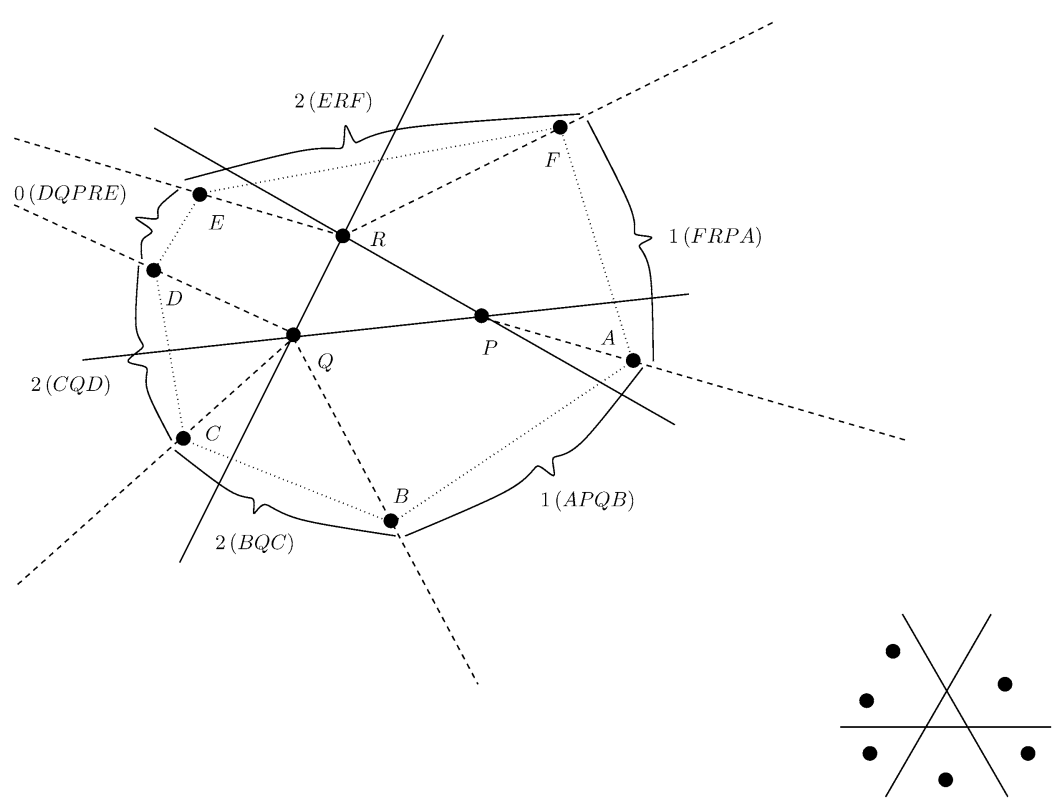

Fig. 8 The case $(6,3,0)$ with $(1,1,1,2,0,1)$
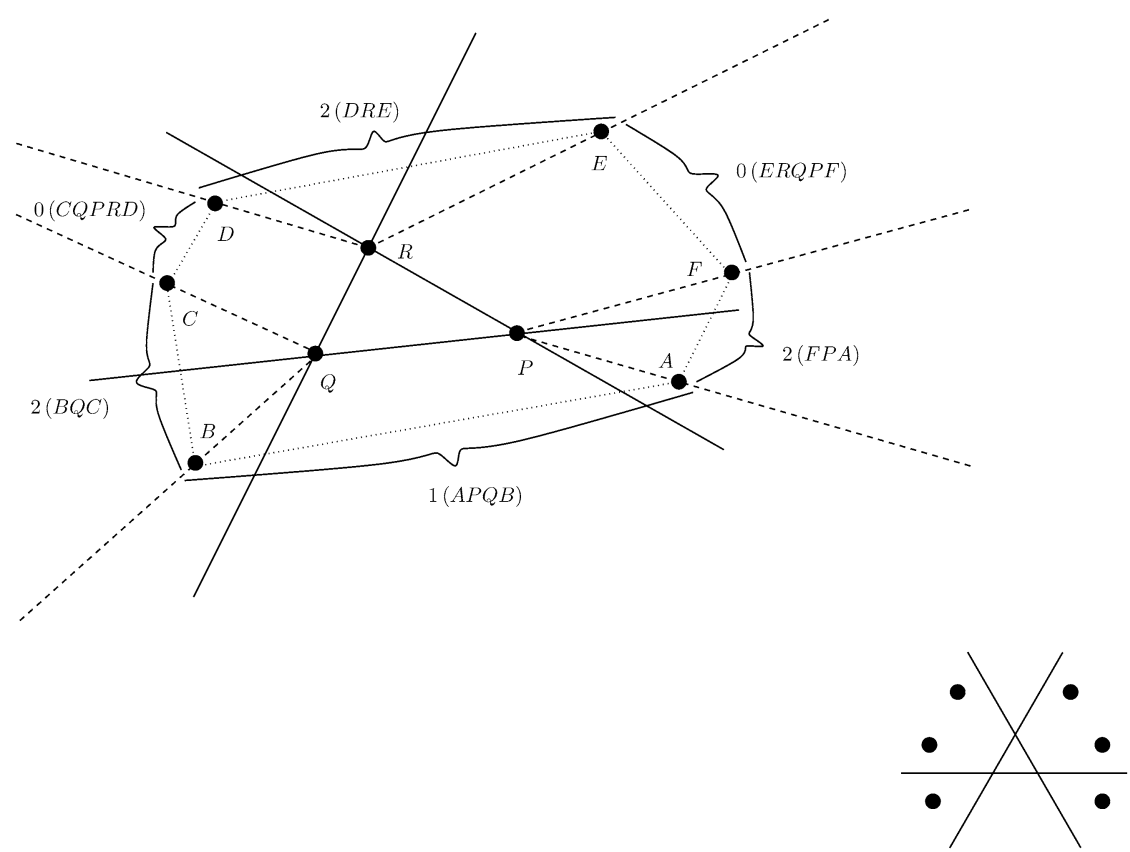

Fig. 9 The case $(6,3,0)$ with $(1,0,1,2,0,2)$ 
Fig. 10 Notation for the cases $(4, \geq 0)$ and $(\geq 7,4)$

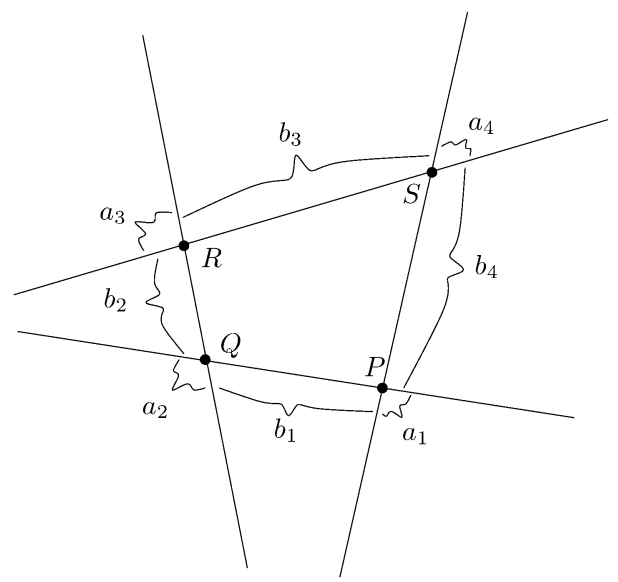

\subsection{Step 1a}

First, consider the cases $(\mathbf{4 , 0})$ and $(\mathbf{8 , 4 , 0 )}$. We use the same type of approach as in Sect. 4. Following the notation as indicated in Fig. 10, where variables again refer to the number of vertices of the 9- respectively 8-gon lying in each sector, we arrive at the set of inequalities

$$
\begin{aligned}
& 1 \leq a_{1}+b_{1}+a_{2} \leq 3, \\
& 1 \leq a_{3}+b_{3}+a_{4} \leq 3,
\end{aligned}
$$

if no empty convex hexagon is to occur. (Vertices lying in more than one sector are assigned arbitrarily to one particular sector they lie in and therefore only counted once.) If no empty convex hexagon is to be present, the constraint

$$
0 \leq b_{2}+b_{4} \leq 1
$$

must also hold. By summing up the upper bounds in (5.1-5.3), it follows that at most seven vertices can be placed around the 4-gon, a contradiction in these two cases.

\subsection{Step $1 b$}

We next consider the case $(\mathbf{7 , 4 , 0 )}$ and evaluate the feasible solutions to the set constraints (5.1-5.3). By symmetry, any feasible $\left(a_{1}, b_{1}, a_{2}, b_{2}, a_{3}, b_{3}, a_{4}, b_{4}\right)$-tuple must also satisfy the following set of inequalities:

$$
\begin{aligned}
& 1 \leq a_{1}+b_{4}+a_{4} \leq 3, \\
& 1 \leq a_{2}+b_{2}+a_{3} \leq 3, \\
& 0 \leq b_{1}+b_{3} \leq 1 .
\end{aligned}
$$

It follows directly from (5.3) and (5.6) that $\sum_{i=1}^{4} b_{i} \leq 2$. Furthermore, it follows from (5.1) and (5.2) (respectively (5.4) and (5.5)) that if $b_{2}=b_{4}=0$ or $b_{1}=b_{3}=0$, 


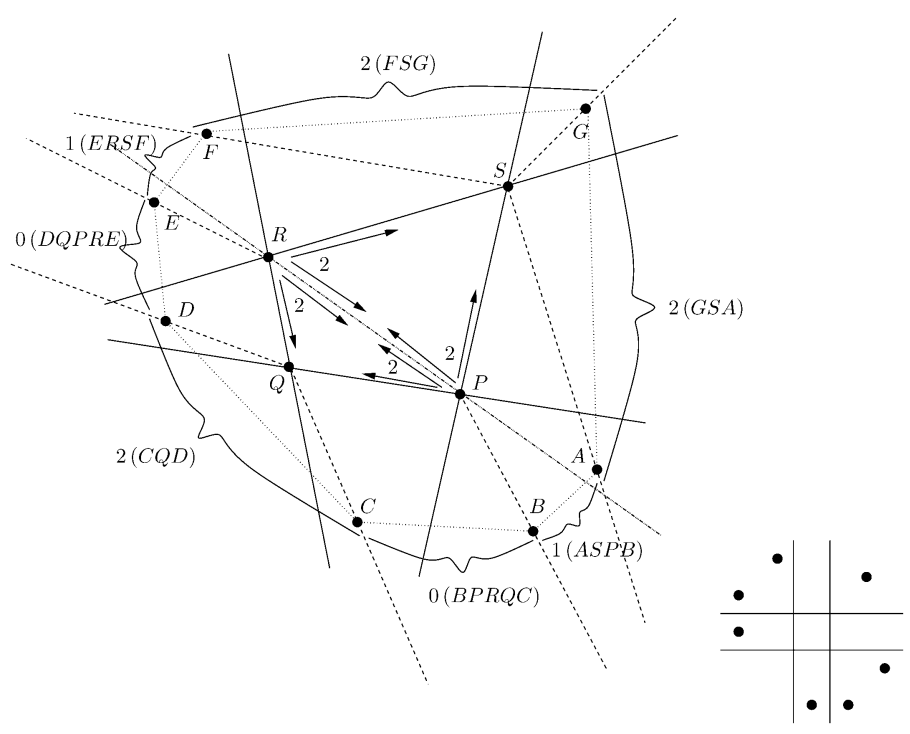

Fig. 11 The case $(7,4,0)$ with $(2,1,0,1,2,0,1,0)$

at most six vertices can be placed around the 4-gon. Therefore, $\left(b_{1}, b_{2}, b_{3}, b_{4}\right)=$ $(1,1,0,0)$ without loss of generality. By choosing $a_{1} \in\{0,1,2\}$, it follows that only the following $\left(a_{1}, b_{1}, a_{2}, b_{2}, a_{3}, b_{3}, a_{4}, b_{4}\right)$-tuples are feasible: $(2,1,0,1,2,0,1,0)$, $(1,1,1,1,1,0,2,0)$ and $(0,1,2,1,0,0,3,0)$ (modulo rotations and reflections). These can be treated individually as follows:

- The subcase $(2,1,0,1,2,0,1,0)$ can be treated as indicated in Fig. 11. Note that at most two of the points $D, E, F$ can lie in one of the sectors $(Q P R)$ and $(R P S)$ without the occurrence of an empty convex hexagon. The same holds for $A, B, C$ and the sectors $(Q R P)$ and $(P R S)$. This is indicated by the arrows. Note that one 4- and one 5-sector arise.

- Figure 12 indicates how to settle the subcase $(1,1,1,1,1,0,2,0)$, provided that the vertex $Q$ of the quadrilateral $P Q R S$ lies outside the triangle $B C D$. In that case, the quadrilateral $B Q D C$ exists. Note that if $Q$ lies inside the triangle $B C D$, there exists an empty convex hexagon $B Q D R S P$.

- The subcase $(0,1,2,1,0,0,3,0)$ can be treated as indicated in Fig. 13. Note that if $B$ and $C$ both lie in $(P S Q)$ or both lie in $(Q S R)$, an empty convex hexagon occurs ( $A B C Q S P$ and $B C D R S Q$ respectively). Again, this is indicated by the arrows.

\subsection{Step 2}

Now we investigate the cases $(4, \mathbf{1})$ and $(\mathbf{8 , 4 , 1 )}$. Consider the sectors occurring when rays emanate from the single point in $J$ (respectively $K$ ) through the vertices of the convex 4-gon. Each of the four sectors can only contain two vertices of the convex 9- respectively 8 -gon as otherwise an empty convex hexagon could be constructed. Since $4 \cdot 2<9$, in the case of the 9-gon an empty convex hexagon must occur. The case of the 8-gon is settled with a similar sector argument on the next level as indicated in Fig. 14. 


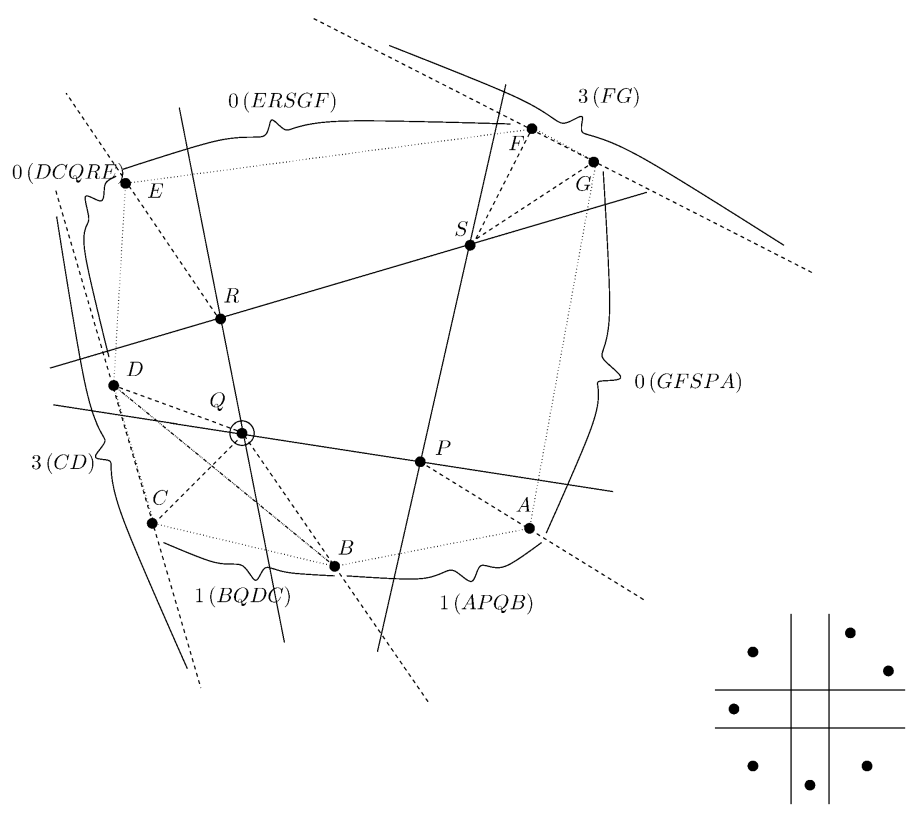

Fig. 12 The case $(7,4,0)$ with $(1,1,1,1,1,0,2,0)$. It is assumed that $Q \notin \triangle B C D$

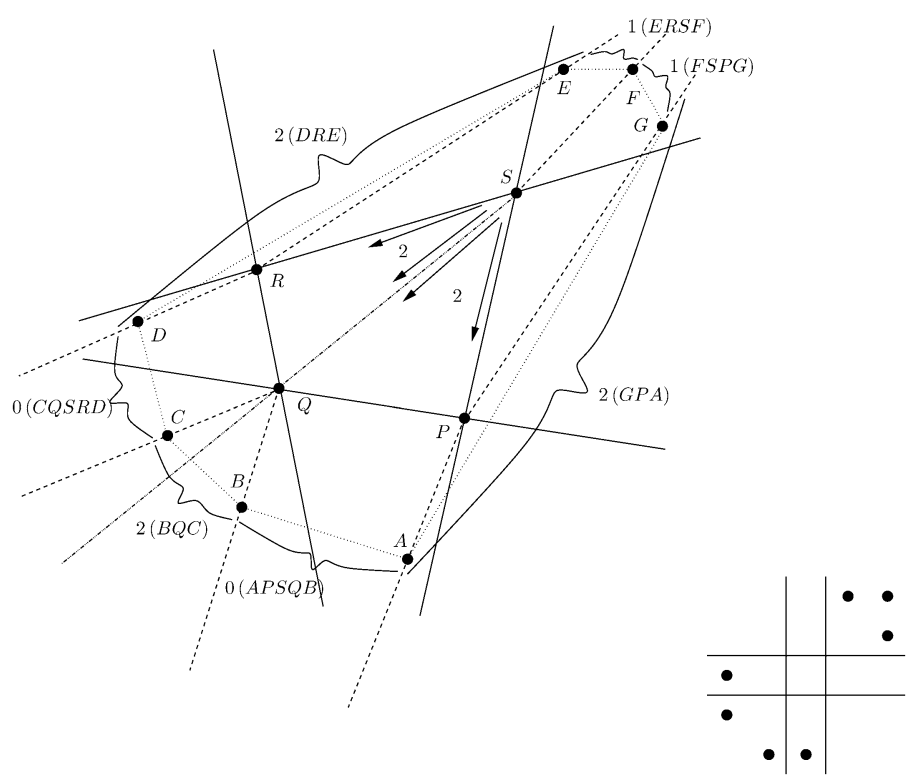

Fig. 13 The case $(7,4,0)$ with $(0,1,2,1,0,0,3,0)$ 


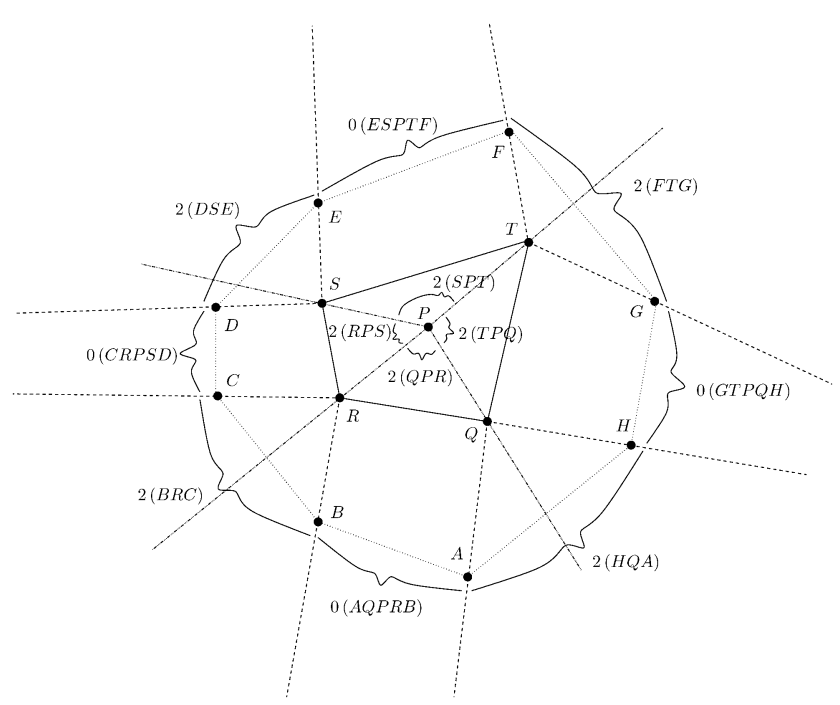

Fig. 14 The case $(8,4,1)$

\subsection{Step 3}

In dealing with the cases $(\mathbf{4}, \geq 2)$, fix a point $P \in J$, construct the sectors as in Step 2 and afterwards replace $P$ with an appropriate point from $J$ in each sector (if necessary). Now argue as in Step 2. Proceed accordingly in the cases $(\mathbf{8 , 4}, \geq \mathbf{2})$ by choosing an arbitrary $P \in K$.

\subsection{Remark}

The approach of Sects. 5.3 and 5.4 also works straightforwardly in the cases $(\mathbf{6}, \mathbf{3}, \geq \mathbf{1})$ (as indicated in Fig. 15), $(\mathbf{7}, \mathbf{3}, \geq \mathbf{1})$ and $(\mathbf{7}, \mathbf{4}, \geq \mathbf{1})$ (as indicated in Fig. 16). Again, the idea is to fix a point $P \in K$ and to create sectors from rays emanating from $P$ that pass through the vertices of the $j$-gon. Argue that each of these sectors can only contain at most two vertices of the $i$-gon without the occurrence of an empty convex hexagon. This remains true if other points of $K$ should lie in some of the sectors. Now create another set of sectors such that their union covers the complete region outside of $\operatorname{conv}(I)$ as indicated in the figures. This approach is extended in Sect. 10 dealing with the cases $(\geq 7, \geq 5, \geq 1)$.

\section{The Cases $(5,0)$ and $(\geq 7,5,0)$}

6.1 The cases $(5,0)$ and $(8,5,0)$

We use the same basic approach as in Sects. 4 and 5, extending the concept and notation of Figs. 3 and 10 in the natural way. We arrive at the set of inequalities

$$
\begin{aligned}
& b_{i}=0 \quad(1 \leq i \leq 5) \quad \text { and } \\
& 1 \leq a_{i}+a_{i+1} \leq 3 \quad\left(1 \leq i \leq 5, a_{6}:=a_{1}\right)
\end{aligned}
$$


Fig. 15 The case $(6,3,1)$

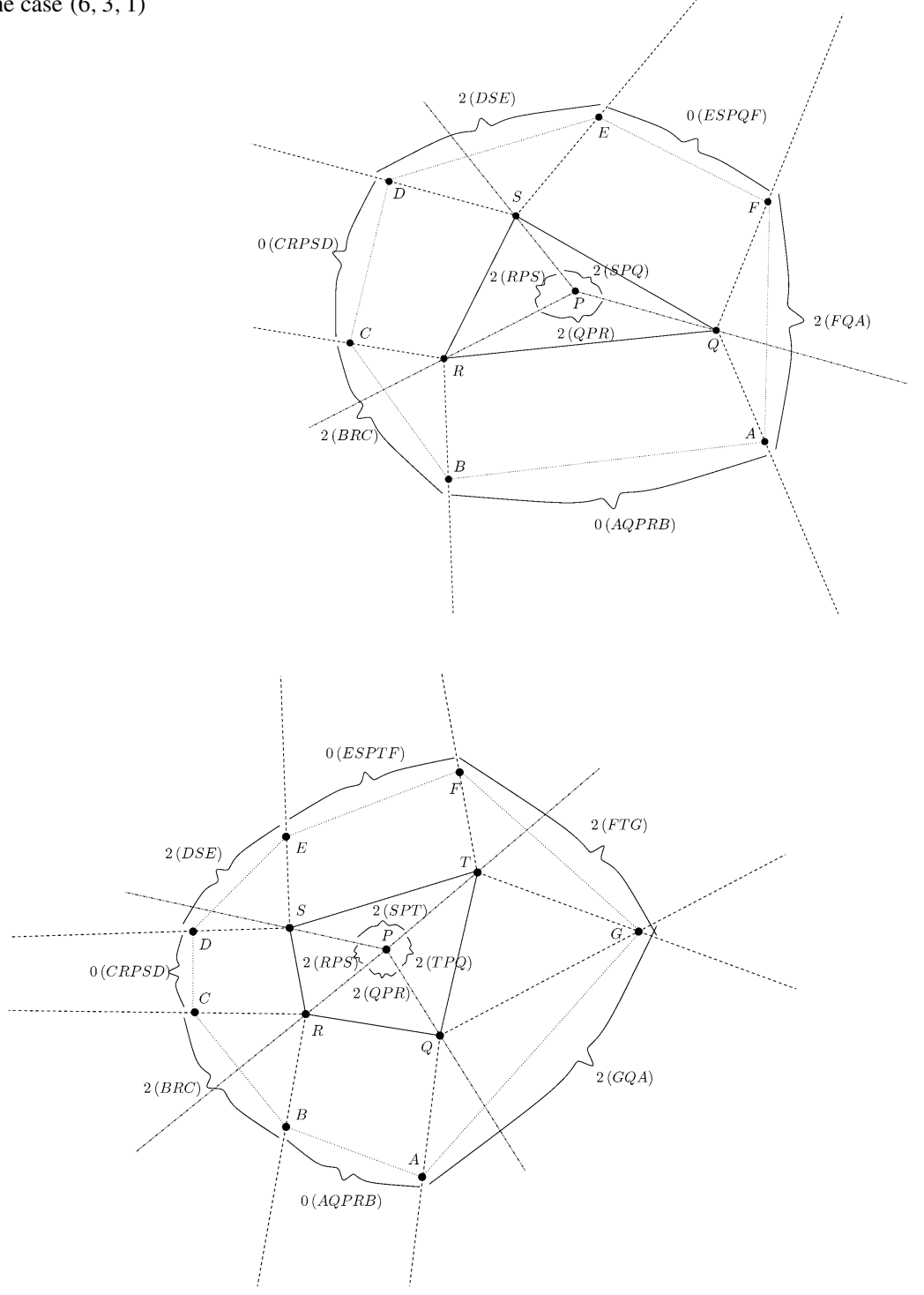

Fig. 16 The case $(7,4,1)$

if no empty convex hexagon is to be present (again counting vertices lying in more than one sector only once). This set of inequalities yields

$$
2 \cdot \sum_{i=1}^{5}\left(a_{i}+b_{i}\right) \leq 15
$$

which implies the desired contradiction that an outer convex polygon with at most seven vertices can be present. 


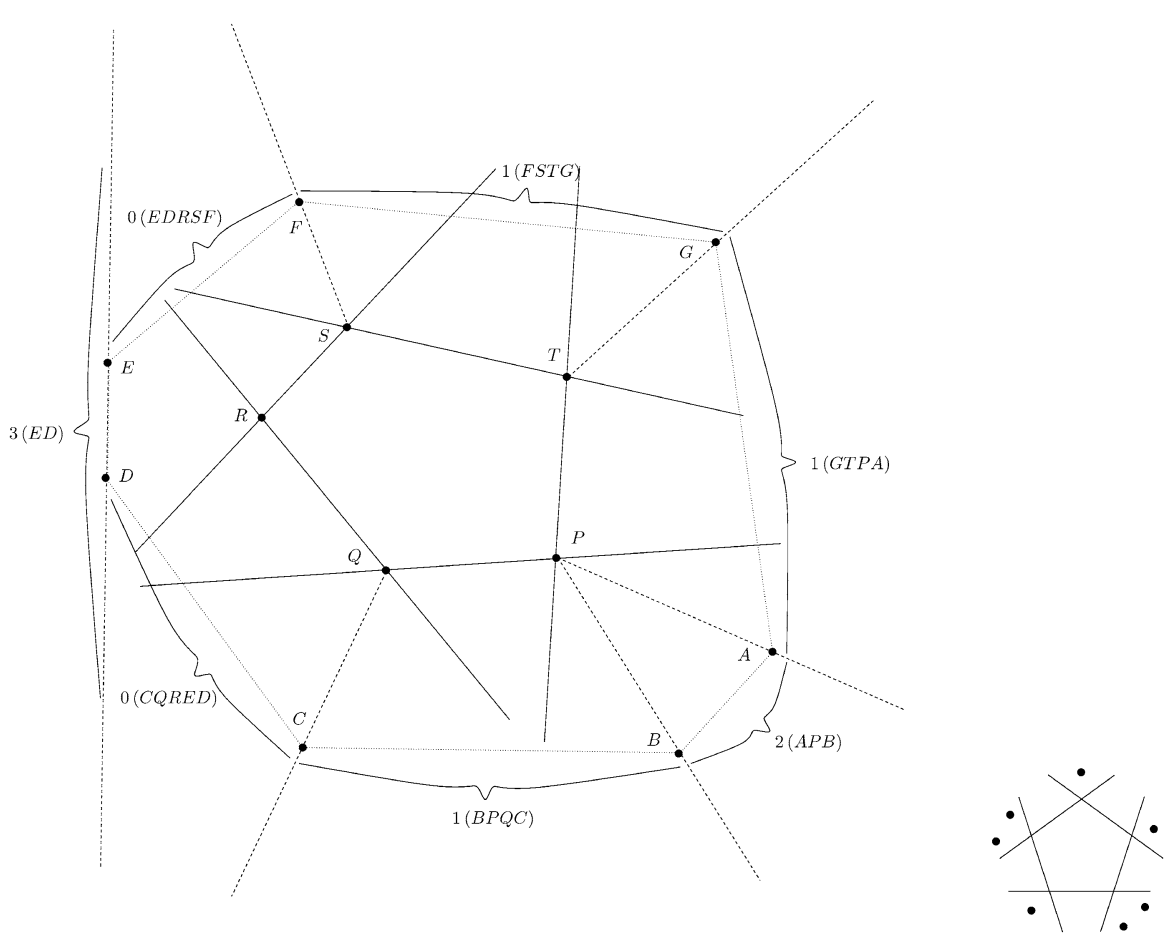

Fig. 17 The case $(7,5,0)$

\subsection{The Case $(7,5,0)$}

A closer investigation of the constraints (6.1-6.3) shows that in this case the only feasible $\left(a_{1}, b_{1}, a_{2}, b_{2}, a_{3}, b_{3}, a_{4}, b_{4}, a_{5}, b_{5}\right)$-tuple (modulo rotation) is (2, 0, 1, 0, 2, 0 , 1, 0, 1,0). This case can be settled as indicated in Fig. 17.

\section{Individual Cases}

\subsection{The Case $(5,1)$}

This case can be dealt with as indicated in Fig. 18. Observe that $P$ must lie in one of the triangles $\triangle A B D, \triangle B C E, \triangle C D A, \triangle D E B$ or $\triangle E A C$ (as these cover the convex 5-gon). Without loss of generality $P$ is inside the triangle $A B D$ (as in the figure). The line $P D$ cuts the 5-gon into the two quadrilaterals $A E D P$ and $P D C B$ (and one triangle). It follows that $m_{1}+m_{2} \leq 1$ and $n_{1}+n_{2} \leq 1$ if no empty convex hexagon is to be present. (As in previous sections, variables refer to the number of vertices of the 9-gon lying in the corresponding sectors.) This leads to at most eight points that can be placed in convex position around the 5-gon without creating an empty convex hexagon. 
Fig. 18 The case $(5,1)$

Fig. 19 The case $(6,1)$. See Sect. 7.2 for details
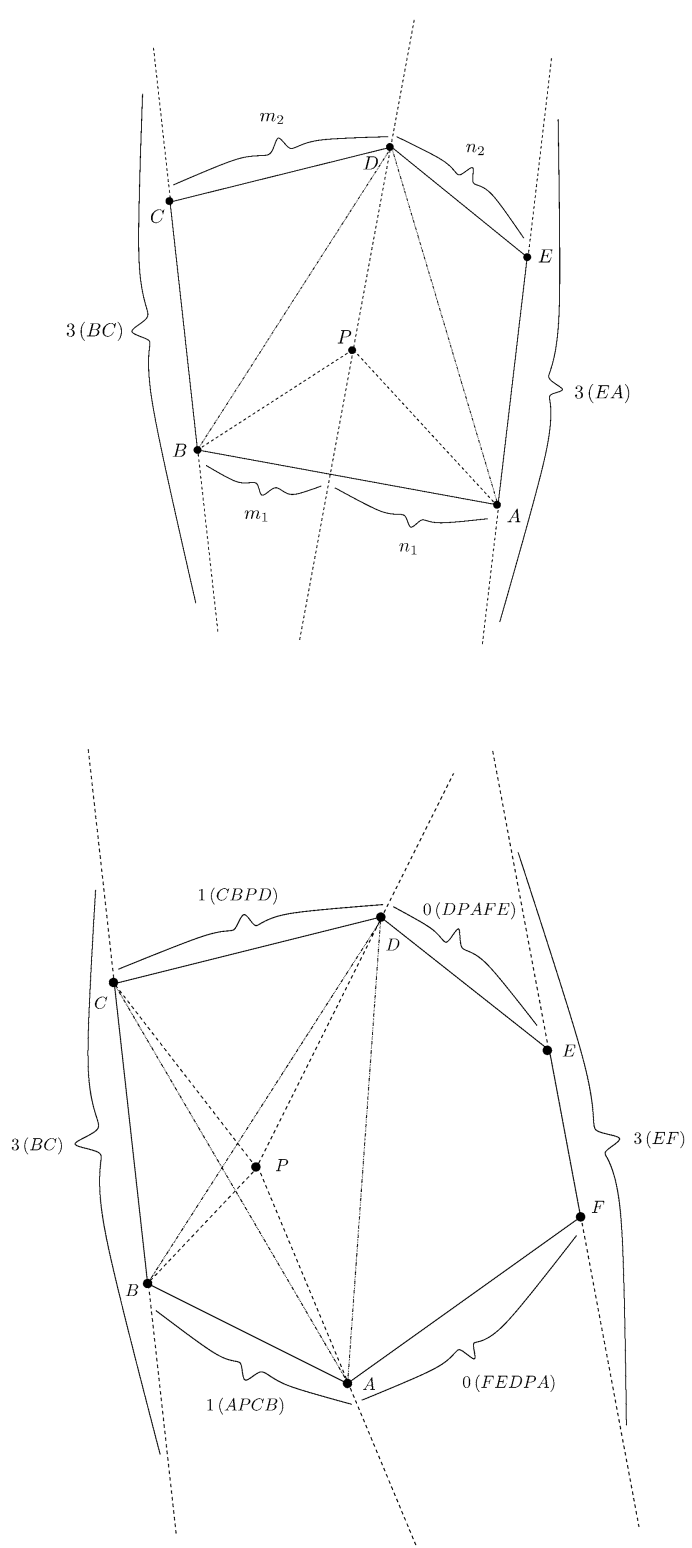

7.2 The Case $(6,1)$

This case can be dealt with as indicated in Fig. 19. Note that $P$ must lie in one of the 4-gons $A D E F$ or $A B C D$ (as in the figure). Note furthermore that if in the latter case, $P \in \triangle A B C$ or $P \in \triangle B C D$, an empty convex hexagon occurs ( $A P C D E F$ respectively $B P D E F A$ ). Therefore, assume that the convex 4-gons $A P C B$ and $C B P D$ exist and argue as indicated in the figure. 
Fig. 20 The case $(6,2)$.

See Sect. 7.3 for details

\subsection{The Cases $(6,2)$ and $(7,1)$}

The case $(\mathbf{6}, \mathbf{2})$ can be dealt with as indicated in Fig. 20. Note that if four vertices of the 6-gon lie on one side of the line $\overline{P Q}$, an empty convex hexagon can be constructed. The case $(\mathbf{7}, \mathbf{1})$ is treated similarly. Here, one of the vertices of the convex 7-gon takes the role of $P$.

\section{The Cases $(5, \geq 2)$}

\subsection{A Key Observation}

The following observation is needed in later sections.

Observation 1 Suppose that $j>2$ and let $2 \leq t \leq \min \{i-1, j\}$. Consider a sequence of $t$ consecutive vertices $V_{1}, V_{2}, \ldots, V_{t}$ of $\operatorname{conv}(J)$. Denote by $T_{n}$ the set of vertices of the i-gon conv $(I)$ lying in the halfplane that is defined by the line $\overline{V_{n} V_{n+1}}$ and that does not contain any other points of $J$. If $\left|\bigcup_{n=1}^{t-1} T_{n}\right|<t$, a 9-gon $H^{\prime}$ with smaller $\left|X \cap \operatorname{conv}\left(H^{\prime}\right)\right|$ can be constructed.

Proof We prove by induction over $t$. We use $U_{l}\left(l \in \mathbb{N}_{0}\right)$ to denote vertices of $\operatorname{conv}(I)$. Note that $\left|T_{n}\right|>0$ for all $n$ by the definition of $J$.

Let $t=2$. Assume that $T_{1}=:\left\{U_{1}\right\}$; see Fig. 21. We claim that at most four vertices of the 9-gon can lie in the union of the 3-sectors $\left(U_{0} V_{1} U_{1}\right)$ and $\left(U_{1} V_{2} U_{2}\right)$, where $U_{0}$ and $U_{2}$ are the vertices of $\operatorname{conv}(\mathrm{I})$ preceding and succeeding $U_{1}$. (Note that $U_{0} \neq U_{2}$ as we presume $t<i$.) The bound follows directly if no other point of $J$ lies within the triangles $\triangle U_{0} V_{1} U_{1}$ respectively $\triangle U_{1} V_{2} U_{2}$. Otherwise replace $V_{1}$ (respectively $V_{2}$ ) by appropriate $V_{1}^{\prime} \in J \cap \triangle U_{0} V_{1} U_{1}$ and $V_{2}^{\prime} \in J \cap \triangle U_{1} V_{2} U_{2}$ to obtain new 


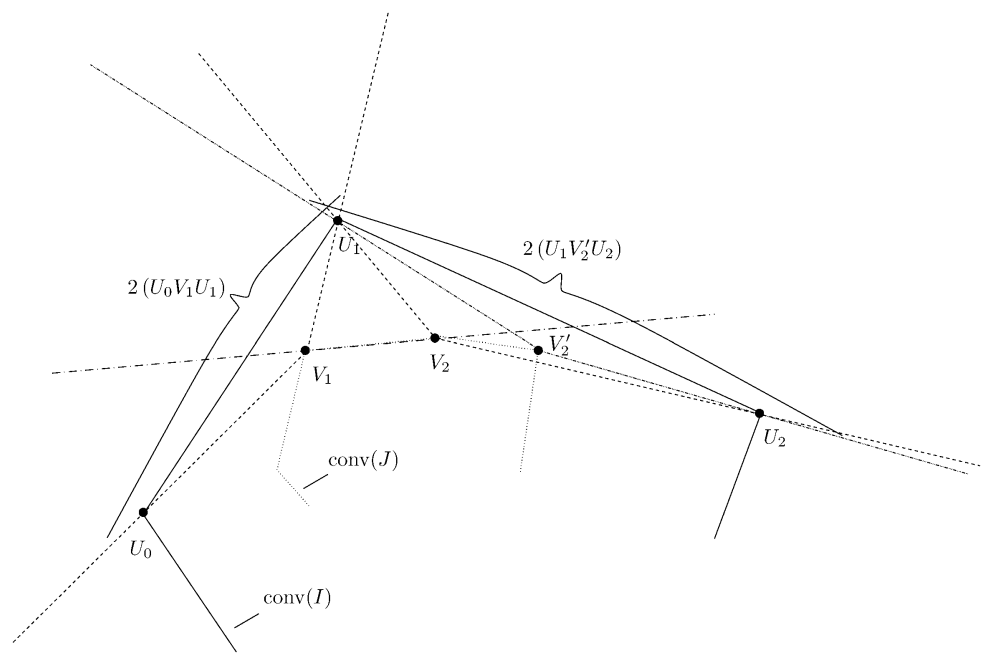

Fig. 21 Observation 1: $t=2$

3-sectors $\left(U_{0} V_{1}^{\prime} U_{1}\right)$ and $\left(U_{1} V_{2}^{\prime} U_{2}\right)$ such that the corresponding triangles $\triangle U_{0} V_{1}^{\prime} U_{1}$ and $\triangle U_{1} V_{2}^{\prime} U_{2}$ do not contain any points of $J$. Note that these 3-sectors cover the region outside of $\operatorname{conv}(I)$ that was originally covered by $\left(U_{0} V_{1} U_{1}\right)$ and $\left(U_{1} V_{2} U_{2}\right)$. (In fact, they cover a larger region.) Each of them allows for at most two vertices of the 9-gon without the occurrence of an empty convex hexagon and the claim follows. Replacing these vertices by points from the convex chain $\overline{U_{0} V_{1} V_{2} U_{1}}$ of length four yields a 9-gon $H^{\prime}$ with smaller $\left|X \cap \operatorname{conv}\left(H^{\prime}\right)\right|$. (A similar argument was used in Sect. 4.2.)

Now let $t>2$. We have to prove that if $\left|\bigcup_{n=1}^{t-1} T_{n}\right|<t$, a 9-gon $H^{\prime}$ with smaller $\left|X \cap \operatorname{conv}\left(H^{\prime}\right)\right|$ can be constructed. If $\left|\bigcup_{n=1}^{t-1} T_{n}\right|<t-1$, we are done by the induction hypothesis as $\left|\bigcup_{n=1}^{t-2} T_{n}\right| \leq\left|\bigcup_{n=1}^{t-1} T_{n}\right|$. Therefore, assume that $\left|\bigcup_{n=1}^{t-1} T_{n}\right|=$ $t-1$. Label the consecutive vertices of $\operatorname{conv}(I)$ as $U_{l}\left(l \in \mathbb{N}_{0}\right)$ in such a way that $U_{1} \in T_{1}$ and $U_{0} \notin T_{1}$. By the induction hypothesis this implies $U_{2} \in T_{1}$ as otherwise $\left|T_{1}\right|=1$. Now construct sectors as follows: start with the 3-sector $\left(U_{0} V_{1} U_{1}\right)$ that can hold at most two vertices of the 9-gon without the occurrence of an empty convex hexagon. (As above, replace $V_{1}$ by $V_{1}^{\prime}$ if necessary.) Next construct the 4-sectors $\left(U_{1} V_{1} V_{2} U_{2}\right),\left(U_{2} V_{2} V_{3} U_{3}\right), \ldots,\left(U_{t-2} V_{t-2} V_{t-1} U_{t-1}\right)$ that can hold at most one vertex of the 9-gon each if no empty convex hexagon is to occur; see Fig. 22.

Note that at each step the construction is well-defined by the induction hypothesis. We can construct the 4-sector $\left(U_{1} V_{1} V_{2} U_{2}\right)$ as $U_{1}, U_{2} \in T_{1}$. Assume there exists a smallest $p \in \mathbb{N}$ such that $U_{p} V_{p} V_{p+1} U_{p+1}$ is not a convex quadrilateral. This means that $U_{p} \in\left(\bigcup_{m=1}^{p-1} T_{m}\right) \backslash T_{p}$ or $U_{p+1} \in\left(\bigcup_{m=p+1}^{t-1} T_{m}\right) \backslash T_{p}$. In the first case, this implies $\left|\bigcup_{m=p}^{t-1} T_{m}\right| \leq(t-1)-p$. In the second case, it follows that $\left|\bigcup_{m=1}^{p} T_{m}\right| \leq p$. In both cases, the induction hypothesis implies that a 9-gon $H^{\prime}$ with smaller $\left|X \cap \operatorname{conv}\left(H^{\prime}\right)\right|$ can be constructed.

Therefore, the 4-sectors can be constructed as described. Finally construct the 3 -sector $\left(U_{t-1} V_{t} U_{t}\right)$ that can hold at most two vertices of the 9-gon without the 


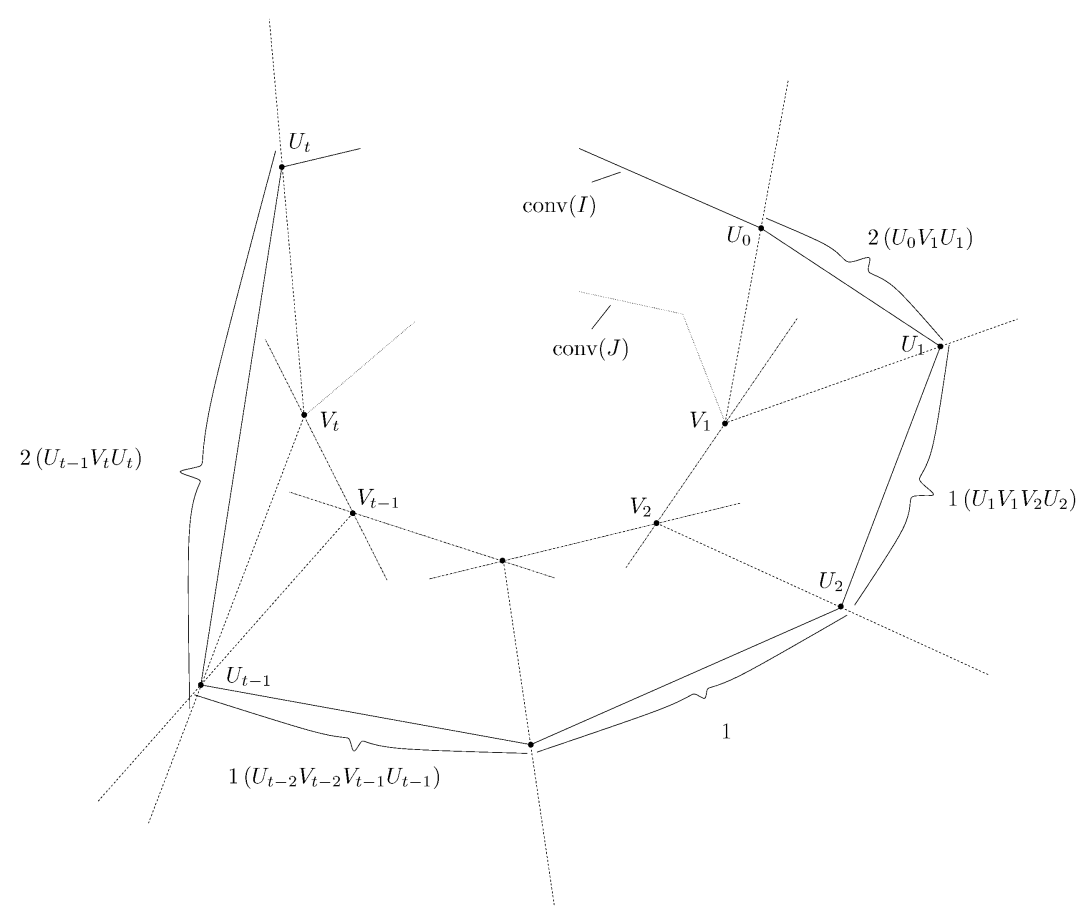

Fig. 22 Observation 1: $t>2$

occurrence of an empty convex hexagon. (As above, replace $V_{t}$ by $V_{t}^{\prime}$ if necessary.) Note that $U_{t} \notin T_{t-1}$ as we presume $\left|\bigcup_{n=1}^{t-1} T_{n}\right|=t-1$. It follows that at most $2 \cdot 2+(t-2) \cdot 1=t+2$ vertices of the 9 -gon can lie in the union of sectors

$$
\left(U_{0} V_{1} U_{1}\right) \cup \bigcup_{l=1}^{t-2}\left(U_{l} V_{l} V_{l+1} U_{l+1}\right) \cup\left(U_{t-1} V_{t} U_{t}\right) \text {. }
$$

Replacing these vertices by points from the convex chain $\overline{U_{0} V_{1} V_{2} \cdots V_{t} U_{t}}$ of length $t+2$ yields a 9-gon $H^{\prime}$ with smaller $\left|X \cap \operatorname{conv}\left(H^{\prime}\right)\right|$.

\subsection{The Cases $(5, \geq 2)$}

Consider the line through two consecutive vertices of $\operatorname{conv}(J)$, say $P$ and $Q$, and let $T_{P Q}$ be the set of vertices of the convex 5-gon lying in a halfplane that is defined by the line $\overline{P Q}$ and that does not contain any other points of $J$. (This halfplane is unique if $|J|>2$.) Consider possible values for $\left|T_{P Q}\right|$ :

- $\left|T_{P Q}\right|=0$ : This case is not possible by the definition of $J$.

- $\left|T_{P Q}\right|=1$ : In this case, a 9-gon $H^{\prime}$ with smaller $\left|X \cap \operatorname{conv}\left(H^{\prime}\right)\right|$ can be constructed. Set $t=2$ in Observation 1 .

- $2 \leq\left|T_{P Q}\right| \leq 3$ : This is the assumption for our subsequent considerations.

- $\left|T_{P Q}\right|>3$ : In this case, an empty convex hexagon can be constructed by using a convex chain of four vertices of the 5-gon together with $P$ and $Q$. 


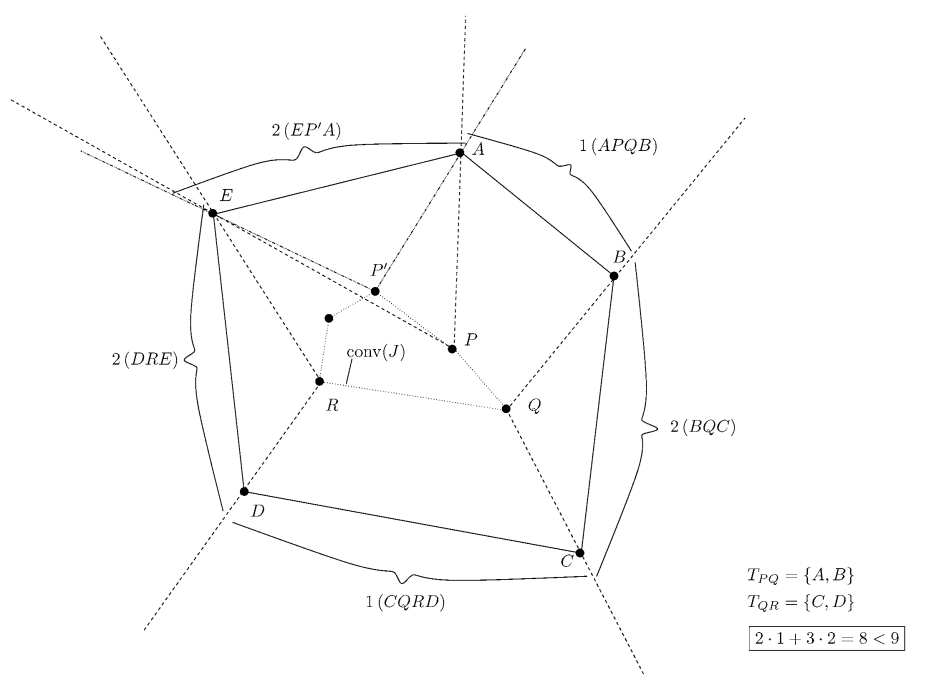

Fig. 23 The cases (5, 2): Example with $\left|T_{P Q} \cup T_{Q R}\right| \geq 4$

Therefore, assume that

$$
2 \leq\left|T_{P Q}\right| \leq 3
$$

Let $R$ be the next vertex on the convex hull of $J$ after passing through $P$ and $Q$ (if $|J|=2$ then $R=P$ ). Define the set $T_{Q R}$ accordingly (take the other halfplane if $|J|=2$ ). For the same reasons as above assume that

$$
2 \leq\left|T_{Q R}\right| \leq 3
$$

and consider the following three possibilities:

\subsection{1 $\left|T_{P Q} \cup T_{Q R}\right| \geq 4$}

In this case we can choose consecutive vertices $A, B, C, D$ of the 5-gon such that $A, B \in T_{P Q}$ and $C, D \in T_{Q R}$. Label the remaining vertex of the 5-gon $E$. Construct the two 4-sectors $(A P Q B)$ and $(C Q R D)$ that can hold at most one vertex of the 9-gon each without the occurrence of an empty convex hexagon. Next construct the 3-sector $(B Q C)$ that can hold at most two vertices of the 9-gon if no empty convex hexagon is to occur. Construct furthermore the two 3-sectors $(D R E)$ and $(E P A)$. Note that the union of these five sectors covers the complete region outside of $\operatorname{conv}(I)$; see also Fig. 23. Each of the two latter 3-sectors can hold at most two vertices of the 9-gon without the occurrence of an empty convex hexagon. (If necessary, replace $R$ (respectively $P$ ) by appropriate $R^{\prime} \in J \cap \triangle D R E$ and $P^{\prime} \in J \cap \triangle E P A$ to obtain new 3-sectors $\left(D R^{\prime} E\right)$ and $\left(E P^{\prime} A\right)$ such that the corresponding triangles $\triangle D R^{\prime} E$ and $\triangle E P^{\prime} A$ do not contain any points of $J$ as in the proof of Observation 1.) It follows that at most $2 \cdot 1+3 \cdot 2=8$ vertices of the 9 -gon can be placed around the 5 -gon without the occurrence of an empty convex hexagon. Note in particular that the case $(\mathbf{5}, \mathbf{2})$ is covered by the argument in this subsection. 


\subsection{2 $\left|T_{P Q} \cup T_{Q R}\right|=3$}

The case $\left|T_{P Q} \cup T_{Q R}\right|=3$ can be treated by the same approach as in the previous subsection. Choose consecutive vertices $A, B, C$ of the 5-gon such that $A, B \in T_{P Q}$ and $B, C \in T_{Q R}$. Label the remaining vertices of the 5-gon $D$ and $E$ such that the vertices $C, D, E$ are consecutive. Construct the two 4-sectors $(A P Q B)$ and $(B Q R C)$. Next construct the 3-sectors $(C R D),(D R E)$ and $(E P A)$. As above, replace the points $R$ and $P$ by appropriate points in $J$ and modify the 3 -sectors if necessary. Again, we arrive at the contradiction that at most $2 \cdot 1+3 \cdot 2=8$ vertices of the 9-gon can be placed around the 5 -gon without the occurrence of an empty convex hexagon.

\subsection{3 $\left|T_{P Q} \cup T_{Q R}\right| \leq 2$}

This case leaves the possibility of constructing a 9-gon $H^{\prime}$ with smaller $\mid X \cap$ $\operatorname{conv}\left(H^{\prime}\right) \mid$. Set $t=3$ in Observation 1.

\section{The Cases $(6, \geq 4)$}

The approach is similar to the one in Sect. 8. The key idea is to partition the region outside of $\operatorname{conv}(I)$ into two 3-sectors and four 4-sectors. Each 3-sector is defined by two consecutive vertices of the 6-gon and one vertex of $\operatorname{conv}(J)$. It can hold at most two vertices of the 9-gon if no empty convex hexagon is to occur. Each 4 -sector is defined by two consecutive vertices of the 6-gon and two consecutive vertices of $\operatorname{conv}(J)$. It can hold at most one vertex of the 9-gon without the occurrence of an empty convex hexagon. It follows that a total of $2 \cdot 2+4 \cdot 1=8$ vertices of the 9-gon can be placed around the 6-gon without the occurrence of an empty convex hexagon.

Consider a chain of consecutive vertices of $\operatorname{conv}(J), \overline{V W X Y Z}$, where $V=Z$ if $j=4$. Define the sets $T_{V W}, T_{W X}, T_{X Y}$ and $T_{Y Z}$ as in Sect. 8 (that is, $T_{V W}$ is the set of vertices of the convex 6-gon lying in the halfplane defined by the line $\overline{V W}$ that does not contain any other points of $J$, etc.). As in Sect. 8, we assume that

$$
2 \leq\left|T_{K L}\right| \leq 3 \quad((K, L) \in\{(V, W),(W, X),(X, Y),(Y, Z)\}) .
$$

By setting $t=3,4,5$ in Observation 1 (Sect. 8), it follows that we may also assume that

$$
\begin{aligned}
& \left|T_{K L} \cup T_{L M}\right| \geq 3, \\
& \left|T_{K L} \cup T_{L M} \cup T_{M N}\right| \geq 4, \\
& \left|T_{V W} \cup T_{W X} \cup T_{X Y} \cup T_{Y Z}\right| \geq 5
\end{aligned}
$$

with $(K, L, M) \in\{(V, W, X),(W, X, Y),(X, Y, Z)\}$ (in (9.2)) and $(K, L, M, N) \in$ $\{(V, W, X, Y),(W, X, Y, Z)\}$ (in (9.3)). Note that $(9.4)$ also holds in the case $(\mathbf{6}, \mathbf{4})$, where Observation 1 does not apply (since $t>j$ ). Note furthermore that by construction it is not possible that there is a $P \in T_{K L} \cap T_{M N}$ with $P \notin T_{L M}((K, L, M, N) \in$ $\{(V, W, X, Y),(W, X, Y, Z)\})$. We now give an explicit construction for the two 3 -sectors and the four 4-sectors. A concrete example can be found in Fig. 24. The combinatorial subcases are depicted in Fig. 25. 


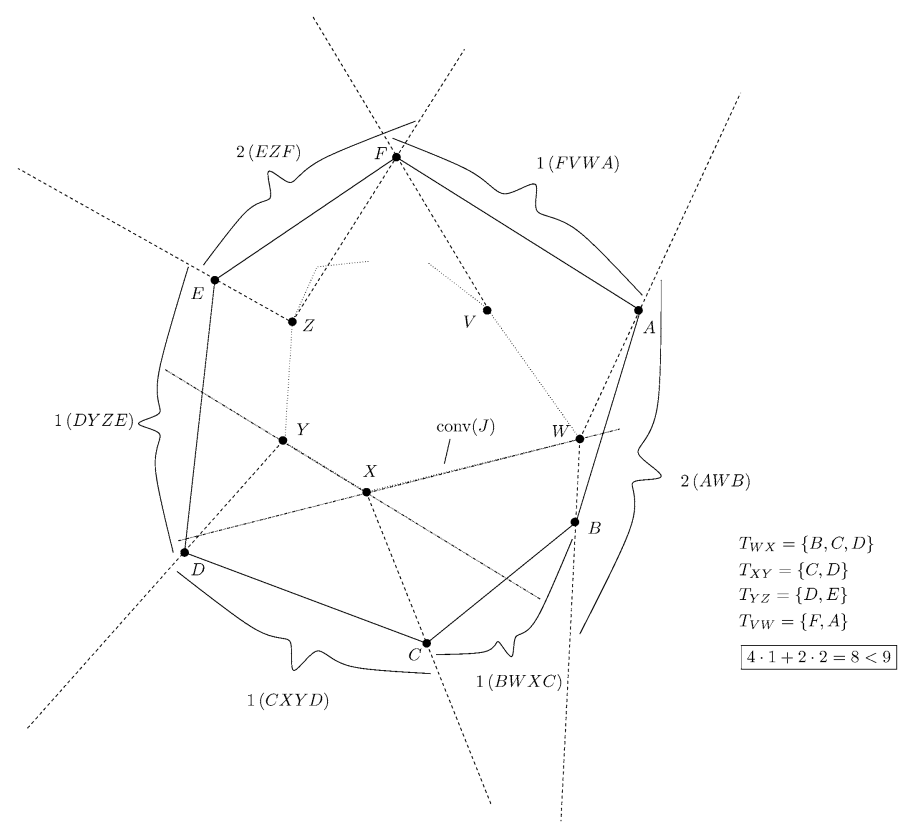

Fig. 24 The cases $(6, \geq 4)$ : Example with $T_{W X} \cap T_{X Y} \neq \emptyset$

\section{$9.1 T_{W X} \cap T_{X Y} \neq \varnothing$}

Label the consecutive vertices of the 6-gon $A, B, C, D, E, F$ such that $B \in T_{W X}, C \in$ $T_{W X} \cap T_{X Y}$ and $D \in T_{X Y}$. Note that $F \notin T_{W X}$ and $F \notin T_{X Y}$ as otherwise $\left|T_{W X}\right|>3$ or $\left|T_{X Y}\right|>3$. Consider the following possibilities:

(1) $A \notin\left(T_{V W} \cup T_{W X}\right)$; see Fig. 25a. It follows from (9.1) and (9.2) that $B, C \in T_{V W}$ and $D \in T_{W X}$. (9.3) implies $E \in T_{X Y}$. Construct the three 4-sectors $(B V W C)$, $(C W X D)$ and $(D X Y E)$. Next, construct the 3-sector $(A V B)$. (Replace $V$ by an appropriate $V^{\prime} \in J \cap \triangle A V B$ if necessary.)

- If $E \in T_{Y Z}$ then (9.4) implies $F \in T_{Y Z}$. Construct the 4-sector ( $\left.E Y Z F\right)$ and the 3 -sector $(F Z A)$. (Again, replace $Z$ by $Z^{\prime}$ if necessary.)

- If $E \notin T_{Y Z}$ then it follows from (9.1) that $A, F \in T_{Y Z}$. ( $F \notin T_{X Y}$ implies in particular $F \notin T_{X Y} \backslash T_{Y Z}$.) In this case construct the 3-sector $(E Y F)$ together with the 4-sector $(F Y Z A)$.

In both cases we arrive at a set of four 4-sectors and two 3-sectors as claimed. In the following cases, assume that $A \in\left(T_{V W} \cup T_{W X}\right)$.

(2) $E \notin\left(T_{X Y} \cup T_{Y Z}\right)$. This case is symmetric to the previous one. Therefore, in the following assume that $E \in\left(T_{X Y} \cup T_{Y Z}\right)$.

(3) $A \in T_{W X} \backslash T_{V W}$; see Fig. 25b. It follows from (9.1) that $E, F \in T_{V W}$. ( $F \notin T_{W X}$ implies in particular that $F \notin T_{W X} \backslash T_{V W}$.) Construct the 3-sectors $(F W A)$ and $(B X C)$ together with the 4-sectors $(E V W F),(A W X B)$ and $(C X Y D)$. It follows that $D \in T_{Y Z}$ as otherwise $\left|T_{Y Z} \cup T_{V W}\right|=|\{E, F\}|<3$. Note that $\left(E \in T_{V W}\right) \wedge\left(E \in\left(T_{X Y} \cup T_{Y Z}\right)\right)$ implies $E \in T_{Y Z}$. Therefore, we 


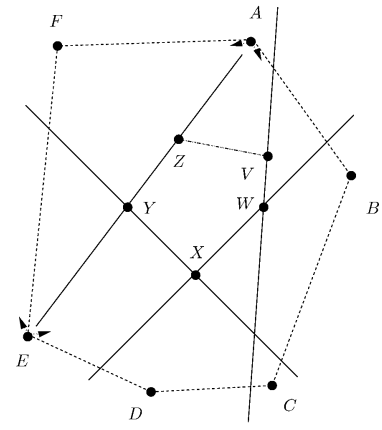

(a)

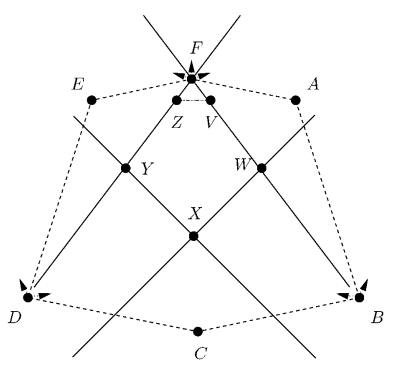

(c)

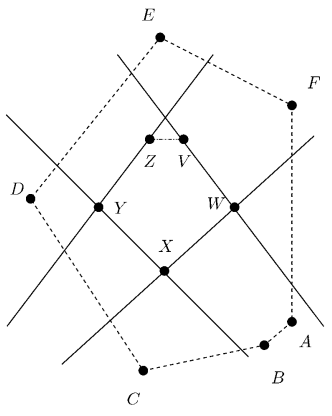

(b)

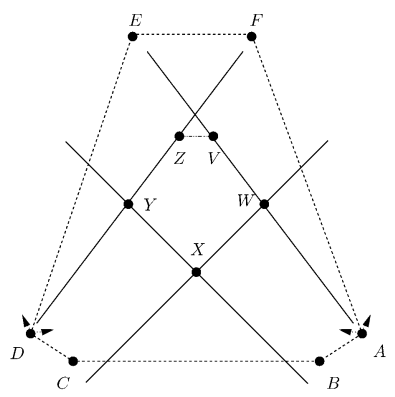

(d)

Fig. 25 The cases $(6, \geq 4)$ : Combinatorial subcases. The assumption in (a)-(c) is that $B \in T_{W X}$, $C \in T_{W X} \cap T_{X Y}$ and $D \in T_{X Y}$. In (a), $A \notin\left(T_{V W} \cup T_{W X}\right)$. In (b), $A \in T_{W X} \backslash T_{V W}$ and $E \in\left(T_{X Y} \cup T_{Y Z}\right)$. In (c), $A \in T_{V W}$ and $E \in T_{Y Z}$. In (d), it is assumed that $A, B \in T_{W X} \backslash T_{X Y}$ and $C, D \in T_{X Y} \backslash T_{W X}$. Only those point positions that are essential for the construction of the sectors are indicated

can construct the 4-sector $(D Y Z E)$. Again the six sectors can be constructed as claimed. In the following assume that $A \in T_{V W}$.

(4) $E \in T_{X Y} \backslash T_{Y Z}$. This case is symmetric to the previous one. Therefore, in the following assume that $E \in T_{Y Z}$.

(5) $A \in T_{V W}$ and $E \in T_{Y Z}$; see Fig. 25c. Construct the 4-sectors ( $B W X C$ ) and $(C X Y D)$. Consider the following four possibilities:

- $B \in T_{V W} \wedge D \in T_{Y Z}$. Construct the 4-sector ( $A V W B$ ) together with the 3 -sector $(F V A)$. (Replace $V$ by $V^{\prime}$ if necessary.) Accordingly, construct the 4-sector $(D Y Z E)$ together with the 3-sector $(E Z F)$. (Replace $Z$ by $Z^{\prime}$ if necessary.)

- $B \notin T_{V W} \wedge D \in T_{Y Z}$. Construct the 4-sector ( $D Y Z E$ ) together with the 3-sector ( $\left.E Z^{\prime} F\right)$ as in the previous subcase. If $B \notin T_{V W}$, it follows from (9.1) that $F \in T_{V W}$. In this case, construct the 3-sector $(A W B)$ together with the 4-sector $(F V W A)$.

- $B \in T_{V W} \wedge D \notin T_{Y Z}$. This subcase is symmetric to the previous one.

- $B \notin T_{V W} \wedge D \notin T_{Y Z}$. It follows that $F \in T_{V W}$ and $F \in T_{Y Z}$. Accordingly, construct the 4-sectors $(F V W A)$ and $(E Y Z F)$ together with the 3-sectors $(A W B)$ and $(D Y E)$. 
In each case, we arrive at a set of four 4-sectors and two 3-sectors that cover the complete region outside of $\operatorname{conv}(I)$ as claimed.

\section{$9.2 T_{W X} \cap T_{X Y}=\emptyset$}

See Fig. 25d. Then by construction, there exist consecutive vertices $A, B, C, D$ of conv(I) such that $A, B \in T_{W X} \backslash T_{X Y}$ and $C, D \in T_{X Y} \backslash T_{W X}$. Construct the 4-sectors $(A W X B)$ and $(C X Y D)$ as well as the 3-sector $(B X C)$. Label the remaining vertices of the 6-gon $E, F$ such that $D, E, F$ are consecutive. Now distinguish four possibilities:

- $D \in T_{Y Z} \wedge A \in T_{V W}$. It follows that $E \in T_{Y Z}$ as otherwise $\left|T_{X Y} \cup T_{Y Z}\right|<3$. Accordingly, $F \in T_{V W}$ as otherwise $\left|T_{V W} \cup T_{W X}\right|<3$. Construct the 4-sectors $(D Y Z E)$ and $(F V W A)$ together with the 3 -sector $(E Z F)$. (Replace $Z$ by an appropriate $Z^{\prime}$ if necessary.)

- $D \notin T_{Y Z} \wedge A \in T_{V W}$. As in the previous case, construct the 4-sector ( $F V W A$ ). If $E \in T_{X Y} \backslash T_{Y Z}$ it follows that $\left|T_{Y Z} \cup T_{V W} \cup T_{W X}\right|=|\{A, B, F\}|<4$. Therefore, assume that $E \in T_{Y Z}$. It follows that $F \in T_{Y Z}$ as otherwise $\left|T_{Y Z}\right|<2$. Construct the 3-sector $(D Y E)$ and the 4-sector $(E Y Z F)$.

- $D \in T_{Y Z} \wedge A \notin T_{V W}$. This case is symmetric to the previous one.

- $D \notin T_{Y Z} \wedge A \notin T_{V W}$. Note that this case is not feasible as it would imply $\mid T_{Y Z} \cup$ $T_{V W}|=|\{E, F\} \mid<3$.

In each feasible case, we can construct the six sectors as claimed above.

\section{The Cases $(\geq 7, \geq 5, \geq 1)$}

Up to this point, we have settled all cases except for $(\geq \mathbf{7}, \geq \mathbf{5}, \geq \mathbf{1})$. These cases, except for three special cases (see below), can all be settled via the same set of arguments. As above, let $K:=\operatorname{conv}(J) \cap(X \backslash \partial J)$. Fix a point $P \in K$. Consider rays emanating from $P$ through each vertex of the convex $j$-gon $\operatorname{conv}(J)$. This divides the region outside the $j$-gon into $j$ sectors and in each sector at most two vertices of $\operatorname{conv}(I)$ can lie without forming an empty convex hexagon. (To see this, construct 3-sectors and replace $P$ by an appropriate $P^{\prime} \in K$ where needed.) Consider all possible vertex distributions. (These are summarized in Table 3.) We want to partition the region outside the convex $i$-gon $\operatorname{conv}(I)$ into sectors and to show that in each case at most eight vertices of the 9-gon can be placed inside the union of these sectors without creating an empty convex hexagon. The following three simple rules are sufficient to prove this:

\subsection{The First Rule}

The first rule deals with two vertices of $\operatorname{conv}(I)$ lying in the same sector.

Rule 1 Let $A_{1}, A_{2}$ denote two consecutive vertices of conv(I) lying in the same sector $(a P b)$, where $a$ and $b$ are consecutive vertices of $\operatorname{conv}(J)$. Then no vertex of the 9-gon can lie in the sector $\left(A_{1} a b A_{2}\right)$ without the occurrence of an empty convex hexagon. 
Table 3 The cases

$(\geq 7, \geq 5, \geq 1)$ : Combinatorial subcases. ( $\Pi$ indicates possible permutations)

\begin{tabular}{ll}
\hline $\mathbf{( 7 , 5 , \geq \mathbf { 1 } )}$ & $\mathbf{( 8 , 5 , \geq \mathbf { 1 } )}$ \\
\hline$\Pi(2,2,2,1,0)$ & $\Pi(2,2,2,2,0)$ \\
$\Pi(2,2,1,1,1)$ & $\Pi(2,2,2,1,1)$ \\
$\mathbf{( 7 , 6 , \geq \mathbf { 1 } )}$ & $\mathbf{( 8 , 6 , \mathbf { 1 } )}$ \\
\hline$\Pi(2,2,2,1,0,0)$ & $\Pi(2,2,2,2,0,0)$ \\
$\Pi(2,2,1,1,1,0)$ & $\Pi(2,2,2,1,1,0)$ \\
$(2,1,1,1,1,1)$ & $\Pi(2,2,1,1,1,1)$ \\
$\mathbf{( 7 , 7 , \geq \mathbf { 1 } )}$ & $\mathbf{( 8 , 7 , \geq \mathbf { 1 } )}$ \\
\hline$\Pi(2,2,2,1,0,0,0)$ & $\Pi(2,2,2,2,0,0,0)$ \\
$\Pi(2,2,1,1,1,0,0)$ & $\Pi(2,2,2,1,1,0,0)$ \\
$\Pi(2,1,1,1,1,1,0)$ & $\Pi(2,2,1,1,1,1,0)$ \\
$(1,1,1,1,1,1,1)$ & $(2,1,1,1,1,1,1)$ \\
$\mathbf{( 7 , 8 , \geq \mathbf { 1 } )}$ & $(\mathbf{8 , 8 , \geq \mathbf { 1 } )}$ \\
\hline$\Pi(2,2,2,1,0,0,0,0)$ & $\Pi(2,2,2,2,0,0,0,0)$ \\
$\Pi(2,2,1,1,1,0,0,0)$ & $\Pi(2,2,2,1,1,0,0,0)$ \\
$\Pi(2,1,1,1,1,1,0,0)$ & $\Pi(2,2,1,1,1,1,0,0)$ \\
$(1,1,1,1,1,1,1,0)$ & $\Pi(2,1,1,1,1,1,1,0)$ \\
& $(1,1,1,1,1,1,1,1)$ \\
\hline
\end{tabular}

Fig. 26 Rule 1

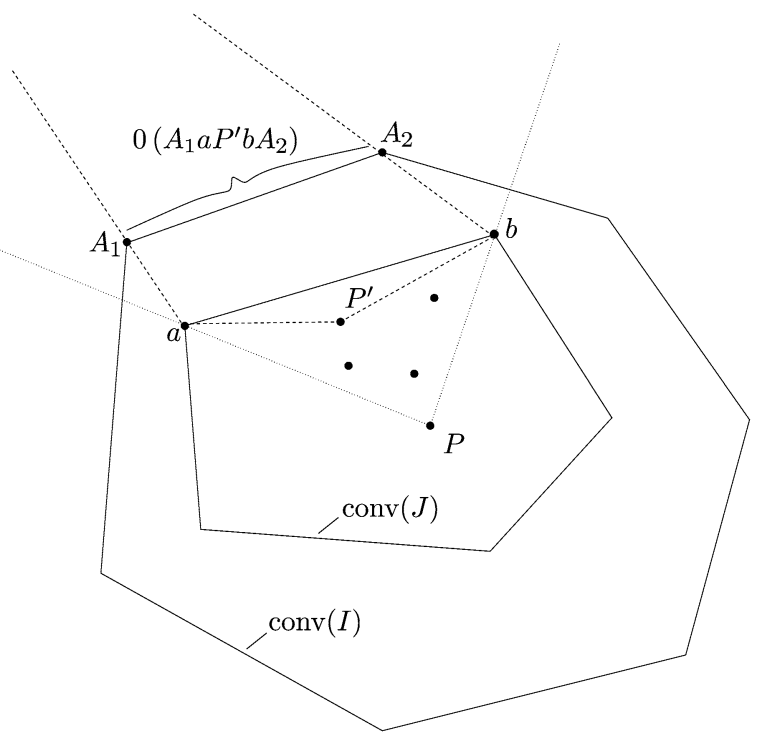

Proof The claim follows directly from the presence of an empty convex 5-gon $A_{1} a P^{\prime} b A_{2}$, where $P^{\prime} \in J \cap \triangle a P b$ is chosen appropriately; see Fig. 26. 
Fig. 27 Rule 2

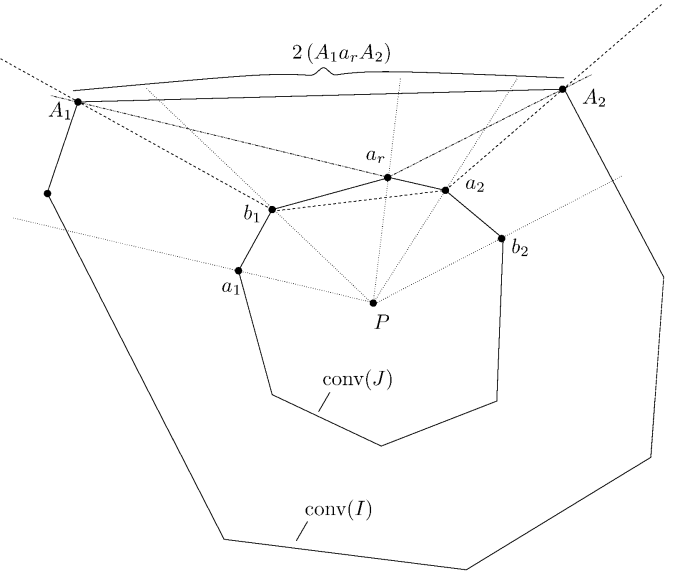

\subsection{The Second Rule}

The second rule gives an upper bound on the number of vertices of the 9-gon that can lie between two non-empty sectors.

Rule 2 Let $A_{1}, A_{2}$ denote two consecutive vertices of conv $(I)$ lying in distinct sectors $\left(a_{1} P b_{1}\right)$ and $\left(a_{2} P b_{2}\right)$, where $a_{1}$ and $b_{1}$ respectively $a_{2}$ and $b_{2}$ are consecutive vertices of $\operatorname{conv}(J)$. Suppose that $a_{1}, b_{1}, a_{2}, b_{2}$ are part of a chain of consecutive vertices of $\operatorname{conv}(J)$. Let $\mathcal{S}:=\left(A_{1} b_{1} a_{2} A_{2}\right)$ if $A_{1} b_{1} a_{2} A_{2}$ is a convex quadrilateral and $\mathcal{S}:=\left(A_{1} b_{1} A_{2}\right) \cup\left(A_{1} a_{2} A_{2}\right)$ otherwise. Then at most two vertices of the 9-gon can lie within $\mathcal{S}$.

Remark 1 It is possible in Rule 2 that $b_{1}=a_{2}$.

Proof A 3-sector that does not contain any points of $J$ and covers the region $\mathcal{S}$ can be constructed by choosing $A_{1}, A_{2}$ and an appropriate $a_{r}$ among the consecutive vertices of $\operatorname{conv}(J)$ between $b_{1}$ and $a_{2}$ (inclusively); see Fig. 27.

\subsection{Application of Rules 1 and 2}

The first two rules are already sufficient to settle the cases $(7,5, \geq 1)$ with distributions $\Pi(2,2,2,1,0),(7,6, \geq 1)$ with distributions $\Pi(2,2,2,1,0,0),(7,7, \geq 1)$ with distributions $\Pi(2,2,2,1,0,0,0),(7,8, \geq 1)$ with distributions $\Pi(2,2,2,1,0,0,0,0)$, $(8,5, \geq 1)$ with distributions $\Pi(2,2,2,2,0),(8,6, \geq 1)$ with distributions $\Pi(2,2,2$, $2,0,0),(8,7, \geq 1)$ with distributions $\Pi(2,2,2,2,0,0,0)$ and $(8,8, \geq 1)$ with distributions $\Pi(2,2,2,2,0,0,0,0)$. To see this, apply Rule 1 whenever two consecutive vertices of $\operatorname{conv}(I)$ lie in the same sector. Note that two such vertices correspond to a 2 in the underlying distribution. For consecutive vertices of conv $(I)$ lying in distinct sectors, apply Rule 2 . Note that in the cases at hand, Rule 2 needs to be applied exactly four times as there are always exactly four non-zero entries in the corresponding distribution sequences. It follows that at most $4 \cdot 2=8$ vertices of the 9-gon can be placed without the occurrence of an empty convex hexagon. An example is given in Fig. 28. 


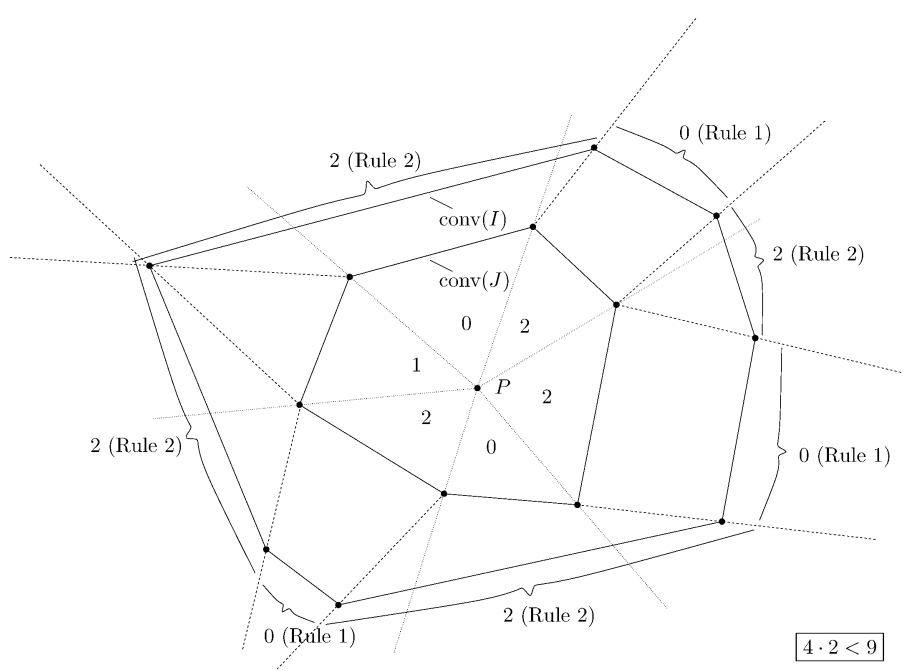

Fig. 28 Application of Rules 1 and 2: Example for the case (7, 6, $\geq 1)$ with distribution $(2,0,2,1,0,2)$

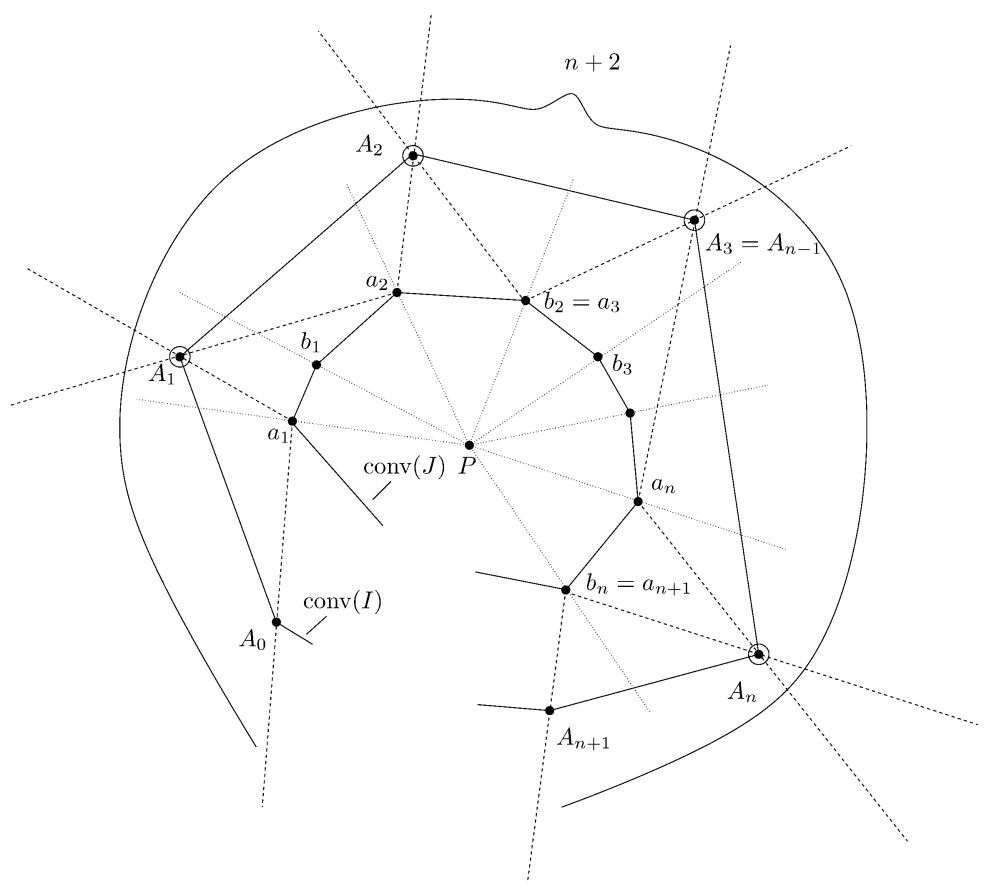

Fig. 29 Rule 3

\subsection{The Third Rule}

The third rule deals with a sequence of sectors, where each sector contains at most one vertex of conv $(I)$. See also Fig. 29. 
Fig. 30 Proof of Rule 3: $n=1$

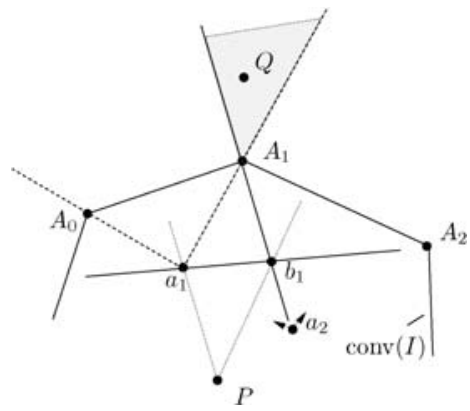

Rule 3 Let $1 \leq n \leq i-2$. Consider a sequence $A_{0}, A_{1}, \ldots, A_{n+1}$ of consecutive vertices of $\operatorname{conv}(I)$. For $1 \leq l \leq n+1$, let $A_{l} \in\left(a_{l} P b_{l}\right)$, where $a_{l}$ and $b_{l}$ are consecutive vertices of $\operatorname{conv}(J)$. Suppose that for $1 \leq l \leq n$, each sector $\left(a_{l} P b_{l}\right)$ contains exactly one vertex of $\operatorname{conv}(I)$ and that $a_{1}, b_{1}, a_{2}, b_{2}, \ldots, a_{n+1}, b_{n+1}$ are part of a chain of consecutive vertices of $\operatorname{conv}(J)$. Then at most $n+2$ vertices of the 9-gon lie in the union of sectors $\bigcup_{l=1}^{n+1}\left(A_{l-1} a_{l} A_{l}\right)$.

Remark 2 It is possible in Rule 3 that $b_{l}=a_{l+1}(1 \leq l \leq n)$ or $b_{n+1}=a_{1}$. Furthermore, it is possible that $A_{0}$ and $A_{n+1}$ both lie in $\left(a_{n+1} P b_{n+1}\right)$.

Proof We prove by induction over $n$.

If $n=1$, we can argue that it is not possible that $A_{1}$ lies above the line $\overline{a_{1} b_{1}}$ while $A_{0}$ and $A_{2}$ lie below it, where lying below refers to lying in the halfplane defined by $\overline{a_{1} b_{1}}$ that includes $P$. Otherwise, $\left|T_{a_{1} b_{1}}\right|=1$ and a 9-gon $H^{\prime}$ with smaller $\left|X \cap \operatorname{conv}\left(H^{\prime}\right)\right|$ could be constructed. (Set $t=2$ in Observation 1 in Sect. 8.) Assume that $A_{0}$ also lies above $\overline{a_{1} b_{1}}$. (The case that only $A_{1}$ and $A_{2}$ lie above the line is similar.) Construct the 4-sector $\left(A_{0} a_{1} b_{1} A_{1}\right)$ together with the 3-sector $\left(A_{1} a_{2} A_{2}\right)$. If necessary, replace $a_{2}$ by an appropriate $a_{2}^{\prime} \in \triangle A_{1} a_{2} A_{2}$ to obtain a new 3-sector $\left(A_{1} a_{2}^{\prime} A_{2}\right)$ with no points of $J$ lying in $\triangle A_{1} a_{2}^{\prime} A_{2}$. Together, the 4 - and the 3 -sector cover (at least) the region of $\left(A_{0} a_{1} A_{1}\right) \cup\left(A_{1} a_{2} A_{2}\right)$. This is clear for points lying in $\left(A_{1} a_{2} A_{2}\right)$ since $\left(A_{1} a_{2}^{\prime} A_{2}\right)$ covers (at least) this region. Note that there cannot be a point $Q \in\left(\left(A_{0} a_{1} A_{1}\right) \backslash\left(A_{1} a_{2}^{\prime} A_{2}\right)\right) \backslash\left(A_{0} a_{1} b_{1} A_{1}\right)$. Such a point would have to lie in the shaded region in Fig. 30. If $a_{2}$ lies to the right of $\overline{b_{1} A_{1}}$ (or $a_{2}=b_{1}$ ) then $Q \in\left(A_{1} a_{2}^{\prime} A_{2}\right)$. Otherwise $b_{1} \in\left(A_{1} a_{2} A_{2}\right)$ and we could have chosen $a_{2}^{\prime}:=b_{1}$. The 4- and the 3 - sector allow for at most $1+2=3$ vertices of the 9-gon without the occurrence of an empty convex hexagon.

For the induction step, assume that the claim is true for $1,2, \ldots, n-1$. By the induction hypothesis, we know that at most $(n-1)+2$ vertices of the 9-gon can lie in the union of sectors $\bigcup_{l=1}^{n}\left(A_{l-1} a_{l} A_{l}\right)$. At most two additional vertices of the 9-gon can lie in the sector $\left(A_{n} a_{n} A_{n+1}\right) \backslash \bigcup_{l=1}^{n}\left(A_{l-1} a_{l} A_{l}\right)$ without the occurrence of an empty convex hexagon as it is part of the 3 -sector $\left(A_{n} a_{n} A_{n+1}\right)$. Therefore, the number of vertices of the 9-gon that can lie in the union of sectors $\bigcup_{l=1}^{n+1}\left(A_{l-1} a_{l} A_{l}\right)$ is at most $(n-1+2)+2=n+3$ if no empty convex hexagon is to occur. It also follows from the induction hypothesis that at most $(n-1)+2$ vertices of the 9 -gon can lie in the union of sectors $\bigcup_{l=2}^{n+1}\left(A_{l-1} a_{l} A_{l}\right)$ without the occurrence of an empty 
convex hexagon. Accordingly, at most two additional vertices of the 9-gon can lie in the sector $\left(A_{0} a_{1} A_{1}\right) \backslash \bigcup_{l=2}^{n+1}\left(A_{l-1} a_{l} A_{l}\right)$ if no empty convex hexagon is to occur. Therefore, the above bound is sharp if and only if exactly two vertices of the 9-gon lie in the sectors $\left(A_{0} a_{1} A_{1}\right)$ and $\left(A_{n} a_{n+1} A_{n+1}\right)$ respectively.

It follows that $A_{0}$ must lie below the line $\overline{a_{1} b_{1}}$ and $A_{n+1}$ must lie below the line $\overline{a_{n} b_{n}}$ as otherwise one could again replace one of the 3 -sectors $\left(A_{0} a_{1} A_{1}\right)$ and $\left(A_{n} a_{n+1} A_{n+1}\right)$ by the 4 -sector $\left(A_{0} a_{1} b_{1} A_{1}\right)$ respectively $\left(A_{n} a_{n} b_{n} A_{n+1}\right)$ as above. This sector could hold only one vertex of the 9-gon (without the occurrence of an empty convex hexagon) and the union of all sectors would still cover the same region.

This implies $\left|\bigcup_{l=1}^{n} T_{a_{l} b_{l}}\right|=n<n+1$, though, and a 9-gon $H^{\prime}$ with smaller $\left|X \cap \operatorname{conv}\left(H^{\prime}\right)\right|$ can be constructed by Observation 1 . To see this, note that $a_{1}, b_{1}, a_{2}, b_{2}, \ldots, a_{n}, b_{n}$ are part of a chain of consecutive vertices of $\operatorname{conv}(J)$ of length $L \geq n+1$. Therefore, the claim follows.

\subsection{Application of Rules 1-3}

Based on the three rules we can now settle all the remaining subcases of $(\geq 7, \geq 5, \geq 1)$ with the exception of $(7,7, \geq 1)$ with distribution $(1,1,1,1,1,1,1)$, $(7,8, \geq 1)$ with distribution $(1,1,1,1,1,1,1,0)$ and $(8,8, \geq 1)$ with distribution $(1,1,1,1,1,1,1,1)$. (These cases do not allow for a direct application of Rule 3 . They are treated individually in the following subsections.) In the other cases, at least one 2 appears in the distribution sequence. We can argue as follows:

Whenever two consecutive vertices of $\operatorname{conv}(I)$ lie within the same sector, apply Rule 1 . Note that two such vertices correspond to a 2 in the underlying distribution. No vertices of the 9-gon can lie in the corresponding sectors.

Now take maximal series of consecutive sectors containing at most one vertex of $\operatorname{conv}(I)$ each and apply Rule 3 (respectively Rule 2 if none of them contains a vertex). The number of vertices of the 9-gon that can lie in the union of all corresponding sectors is equal to $q+s \cdot 2$, where $q$ is the total number of 1 's in the underlying distribution and $s$ is the number of distinct series. Note that $s$ is equal to the number of gaps between two occurrences of a 2 in the distribution sequence. As this number is equal to the number of 2's in the sequence, it follows that $q+s \cdot 2$ is equal to the sum of the elements of the distribution sequence. It can easily be verified that this sum is always smaller than 9. Therefore, in all these cases an empty convex hexagon occurs. (An example is given in Fig. 31.)

\subsection{The Case $(1,1,1,1,1,1,1)$}

This case can be dealt with by applying Rule 3 with $n=5$ seven times with each vertex of $\operatorname{conv}(I)$ as a starting point. Each 3 -sector $\left(A_{r-1} a_{r} A_{r}\right)$ is left out exactly once. Therefore, in the union of all sectors at most $(7 \cdot(5+2)) / 6<9$ vertices of the 9 -gon can lie without the occurrence of an empty convex hexagon.

\subsection{The Case $(1,1,1,1,1,1,1,0)$}

For the case $(1,1,1,1,1,1,1,0)$, label the vertices of the polygon $\operatorname{conv}(J)$ in clockwise order $a_{l}(1 \leq l \leq 8)$ and assume that the sector $\left(a_{6} P a_{7}\right)$ is the one that does not 


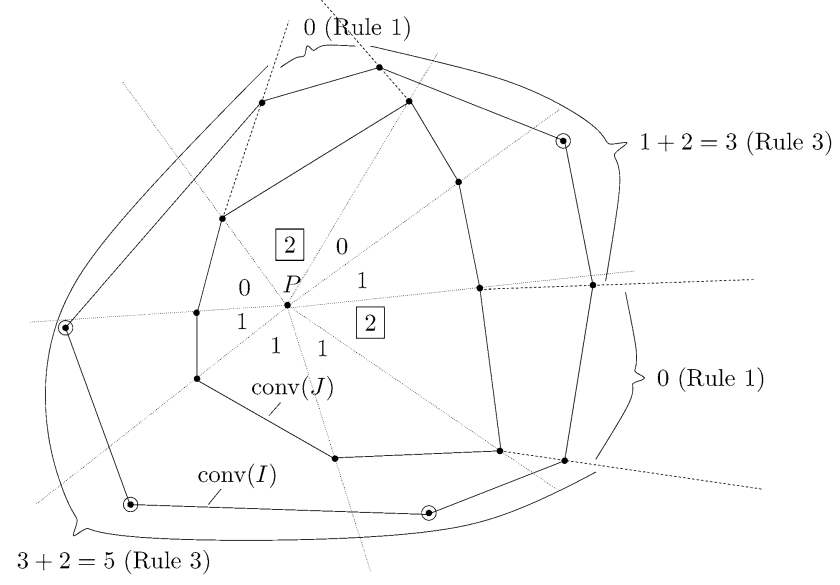

Fig. 31 The case $(8,8, \geq 1)$ with $(2,1,1,1,0,2,0,1)$

Fig. 32 The case $(7,8, \geq 1)$ with $(1,1,1,1,1,1,1,0)$

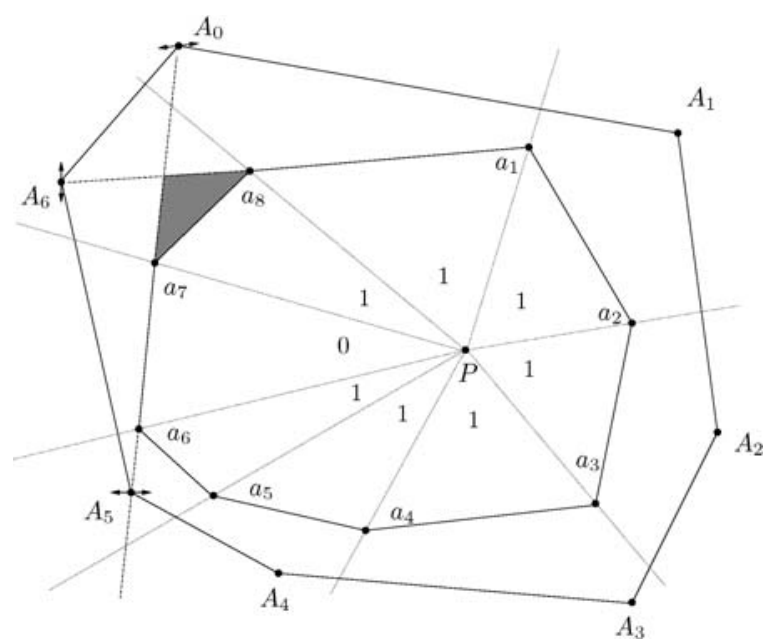

contain a vertex of $\operatorname{conv}(I)$. Applying Rule 3 with $n=5$, we can conclude that at most $5+2=7$ vertices of the 9-gon can lie in the union of sectors $\bigcup_{l=1}^{6}\left(A_{l-1} a_{l} A_{l}\right)$; see Fig. 32. Consider $A_{6}$. Note that it is not possible that $A_{6}$ lies below the line $\overline{a_{1} a_{8}}$ and below the line $\overline{a_{6} a_{7}}$ (where below refers to the halfplane that includes $P$ ), as otherwise a 9-gon $H^{\prime}:=\left(a_{8} a_{1} a_{2} \cdots a_{7} A_{6}\right)$ with smaller $\left|X \cap \operatorname{conv}\left(H^{\prime}\right)\right|$ is present.

If $A_{6}$ lies above the line $\overline{a_{1} a_{8}}$, only one vertex of the 9-gon can lie in the then existing 4-sector $\left(A_{6} a_{8} a_{1} A_{0}\right)$ and therefore, without the occurrence of an empty convex hexagon, at most eight vertices of the 9-gon can lie in the union of sectors

$$
\bigcup_{l=1}^{6}\left(A_{l-1} a_{l} A_{l}\right) \cup\left(A_{6} a_{8} a_{1} A_{0}\right),
$$

which by construction covers the complete region outside of $\operatorname{conv}(I)$. 
Similarly, if $A_{0}$ lies above the line $\overline{a_{6} a_{7}}$ (and therefore also $A_{6}$ by construction), at most eight vertices of the 9-gon can lie in the union of sectors

$$
\bigcup_{l=1}^{6}\left(A_{l-1} a_{l} A_{l}\right) \cup\left(A_{6} a_{6} a_{7} A_{0}\right)
$$

(which by construction covers the complete region outside of $\operatorname{conv}(I)$ ) without the occurrence of an empty convex hexagon.

Finally, if $A_{0}$ lies below the line $\overline{a_{6} a_{7}}$ and $A_{6}$ lies above it, we know that $A_{5}$ must also lie above the line $\overline{a_{6} a_{7}}$ as otherwise $\left|T_{a_{6} a_{7}}\right|<2$ and a 9-gon $H^{\prime}$ with smaller $\left|X \cap \operatorname{conv}\left(H^{\prime}\right)\right|$ could be constructed by Observation 1 . Therefore, the 4sector $\left(A_{5} a_{6} a_{7} A_{6}\right)$ exists, which can only hold one vertex of the 9-gon without the occurrence of an empty convex hexagon. Now, applying Rule 3 with $n=5$ yields that at most seven vertices of the 9-gon can lie in the union of sectors

$$
\left(A_{6} a_{8} A_{0}\right) \cup \bigcup_{l=1}^{5}\left(A_{l-1} a_{l} A_{l}\right)
$$

without the occurrence of an empty convex hexagon. Therefore, without the occurrence of an empty convex hexagon, at most eight vertices of the 9-gon can lie in the union of sectors

$$
\left(A_{6} a_{8} A_{0}\right) \cup \bigcup_{l=1}^{5}\left(A_{l-1} a_{l} A_{l}\right) \cup\left(A_{5} a_{6} a_{7} A_{6}\right)
$$

which by construction covers the complete region outside of conv $(I)$.

10.8 The Case $(1,1,1,1,1,1,1,1)$

Note that in the case $(1,1,1,1,1,1,1,1)$, applying the induction argument with $n=6$ eight times with each vertex of $\operatorname{conv}(J)$ as a starting point (in analogy to our approach to the case $(7,7, \geq 1)$ with distribution $(1,1,1,1,1,1,1)$ in Sect. 10.6) only gives us an estimate of a total of $(8 \cdot(6+2)) / 7>9$ vertices of the 9 -gon that can lie in the union of all sectors. Therefore, a different approach for this subcase is required.

Label the vertices of conv $(J)$ in clockwise order as $a_{r}(1 \leq r \leq 8)$. Consider four consecutive vertices of the convex 8-gon $\operatorname{conv}(J), a_{s}, a_{t}, a_{u}$ and $a_{v}$. Note that no vertex of $\operatorname{conv}(I)$ can lie below the line $\overline{a_{s} a_{t}}$ and below the line $\overline{a_{u} a_{v}}$ (where below refers to the halfplane that includes $P$ ) as otherwise we could use such a vertex to construct a 9-gon $H^{\prime}$ with smaller $\left|X \cap \operatorname{conv}\left(H^{\prime}\right)\right|$. Denote by $R_{t}$ the region above both lines $\overline{a_{s} a_{t}}$ and $\overline{a_{t} a_{u}}$; see Fig. 33. The union of all regions $R_{r}(1 \leq r \leq 8)$ defines the feasible region for vertices of conv $(I)$. Label the vertices of $\operatorname{conv}(I)$ as $A_{m}\left(A_{m} \in\right.$ $\left.\left(a_{m} P a_{m+1}\right), 1 \leq m \leq 8, a_{9}:=a_{1}\right)$. Note that $A_{m}$ lies in $R_{m}$ or $R_{m+1}$ (or both) $(1 \leq$ $\left.m \leq 8, R_{9}:=R_{1}\right)$. Consider the following three possibilities: 


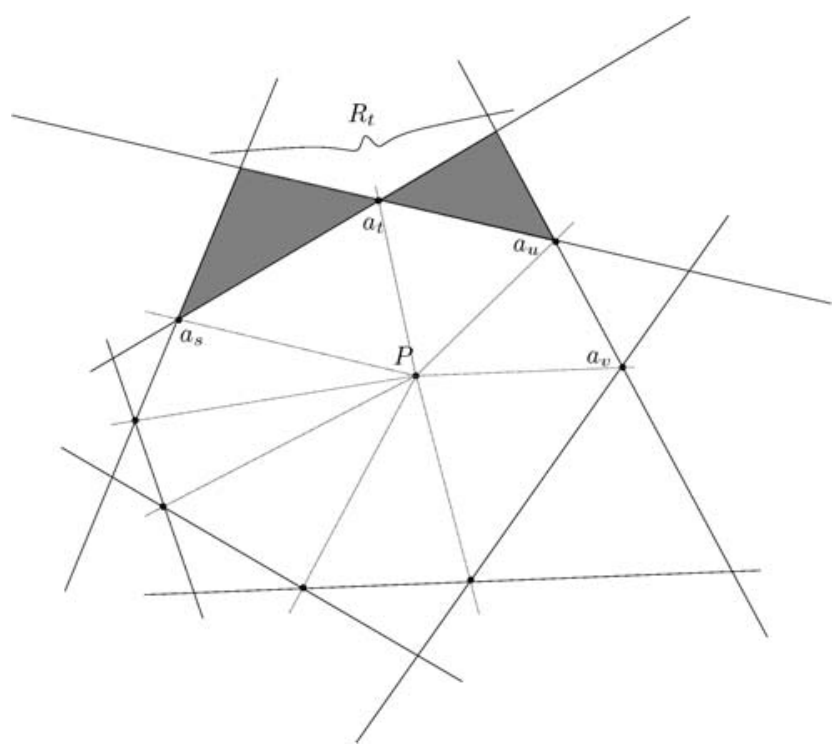

Fig. 33 The case $(8,8, \geq 1)$ with $(1,1,1,1,1,1,1,1)$ : Definition $R_{t}$

\subsubsection{There Exists a Region $R_{w}$ with no $A_{m}$ Lying in It}

There can be at most one such region as otherwise one could construct a 9-gon $H^{\prime}$ with smaller $\left|X \cap \operatorname{conv}\left(H^{\prime}\right)\right|$. To see this, eliminate successively the possibilities that the next $R_{z}$ with this property is $R_{w+1}, R_{w+2}, R_{w+3}$ or $R_{w+4}$. In the first case, $\left|T_{a_{w} a_{w+1}}\right|<2$ and in the other three cases one can replace one to three vertices of the 8-gon $a_{1} a_{2} \cdots a_{8}$ by two to four points $A_{m}$ in such a way that a 9-gon $H^{\prime}$ with smaller $\left|X \cap \operatorname{conv}\left(H^{\prime}\right)\right|$ appears.

Let $a_{1}$ be the vertex associated with the region that does not contain any $A_{m}$. Since the existence of such a region is independent of the choice of $P$, we may assume that $P$ lies in the pentagon $a_{6} a_{2} a_{3} a_{4} a_{5}$. Such a $P$ must exist for otherwise an empty convex hexagon appears; see also Fig. 34. (A different choice of $P$ might result in a different distribution sequence. If this is the case, we arrive at a subcase that has already been settled.) As a consequence, at most three vertices of the 9-gon can lie in the sector $\left(A_{6} a_{6} P a_{2} A_{1}\right)$ as otherwise a 9-gon $H^{\prime}$ with smaller $\left|X \cap \operatorname{conv}\left(H^{\prime}\right)\right|$ could be constructed (as $5+4=9$ ).

We claim that $A_{m} \in R_{m} \cap R_{m+1}$ for $2 \leq m \leq 7$; that is, each $A_{m}$ lies above both lines $\overline{a_{m-1} a_{m}}$ and $\overline{a_{m+1} a_{m+2}}\left(2 \leq m \leq 7, a_{9}:=a_{1}\right)$. To see this, start from the line $\overline{a_{1} a_{2}}$ and work clockwise to prove that $A_{m}$ is above the line $\overline{a_{m-1} a_{m}}(2 \leq m \leq 7)$. Note that configurations, where $\left|\bigcup_{n=1}^{l-1} T_{a_{n} a_{n+1}}\right|<l(2 \leq l \leq 8)$ yield a 9 -gon $H^{\prime}$ with smaller $\left|X \cap \operatorname{conv}\left(H^{\prime}\right)\right|$ by Observation 1 (Sect. 8). Now start from the line $\overline{a_{1} a_{8}}$ and work counter clockwise to prove that $A_{m}$ is above the line $\overline{a_{m+1} a_{m+2}}(7 \geq m \geq 2$, $\left.a_{9}:=a_{1}\right)$.

Finally, as we are assuming that no $A_{r}$ lies in $R_{1}$, it follows that $A_{1} \in R_{2}$ and $A_{8} \in R_{8}$. Therefore, this case can be settled as indicated in Fig. 34. 


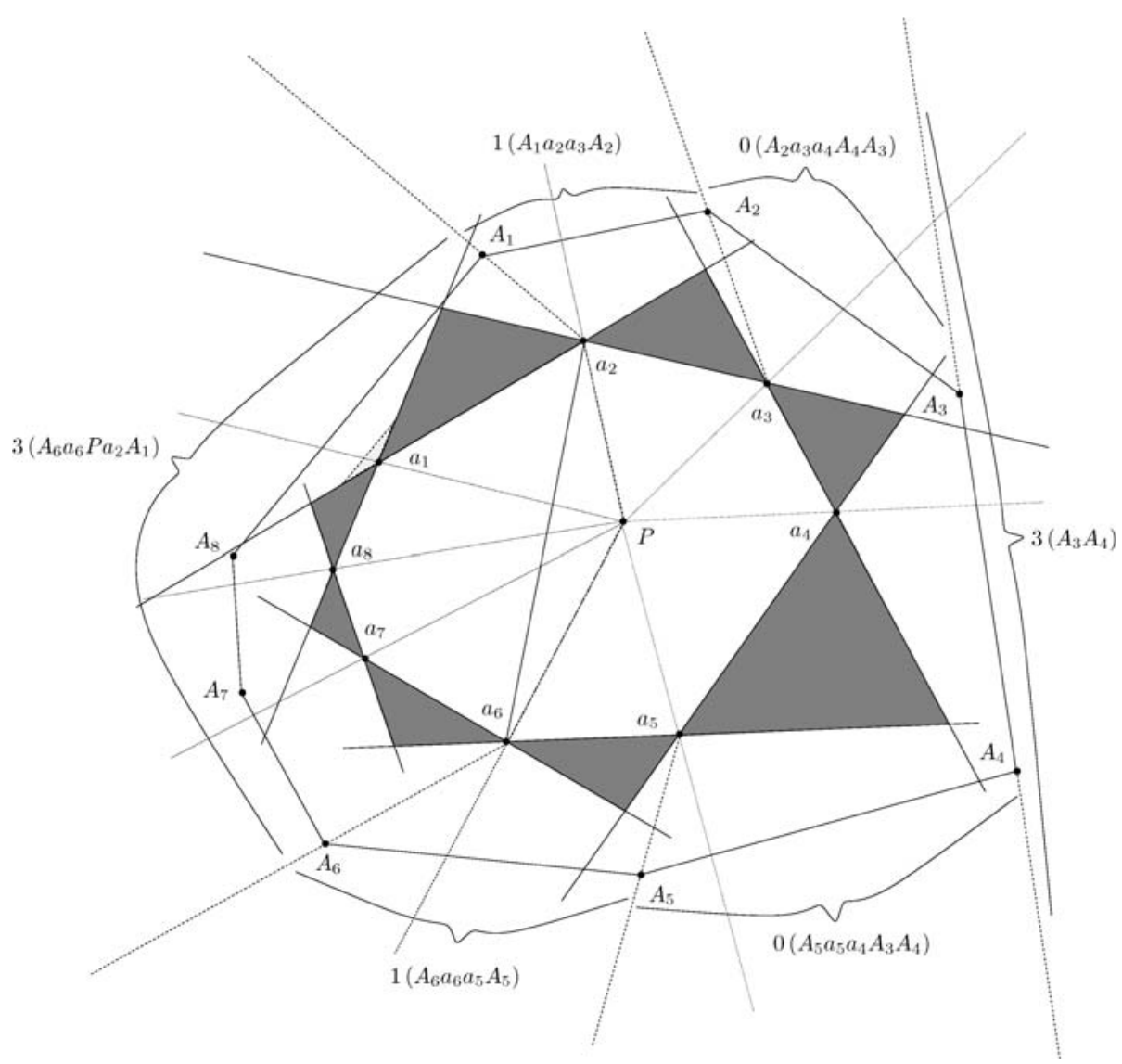

Fig. 34 The case $(8,8, \geq 1)$ with $(1,1,1,1,1,1,1,1): R_{1}$ contains no $A_{m}$

10.8.2 (At Least) One $A_{m}$ Lies in Each Region $R_{r}(1 \leq r \leq 8)$ and, Say, $A_{1} \in R_{1} \backslash R_{2}$

We claim that this implies that $A_{u} \in R_{u}(3 \leq u \leq 8)$ as otherwise a 9-gon $H^{\prime}$ with smaller $\left|X \cap \operatorname{conv}\left(H^{\prime}\right)\right|$ appears. To see this, first consider the point $A_{8}$. If $A_{8} \in$ $R_{1} \backslash R_{8}$, the 9-gon $H^{\prime}:=A_{1} a_{2} a_{3} \cdots a_{8} A_{8}$ with smaller $\left|X \cap \operatorname{conv}\left(H^{\prime}\right)\right|$ occurs. Therefore, $A_{8} \in R_{8}$. Next, consider $A_{7}$, then $A_{6}$ and so on. Finally, as we are assuming that at least one $A_{m}$ lies in each region $R_{r}(1 \leq r \leq 8)$ it follows that $A_{2} \in R_{2}$; see Fig. 35. In this case, the region outside of $\operatorname{conv}(I)$ can be partitioned into eight 4-sectors $\left(A_{l} a_{l} a_{l+1} A_{l+1}\right)\left(1 \leq l \leq 8, a_{9}:=a_{1}, A_{9}:=A_{1}\right)$ that together allow at most eight vertices of the 9-gon without the occurrence of an empty convex hexagon.

\subsubsection{Each $A_{m}$ Lies in Both $R_{m}$ and $R_{m+1}$}

Again, the region outside of $\operatorname{conv}(I)$ can be partitioned into eight 4-sectors $\left(A_{l} a_{l} a_{l+1} A_{l+1}\right)\left(1 \leq l \leq 8, a_{9}:=a_{1}, A_{9}:=A_{1}\right)$ that together allow at most eight vertices of the 9-gon without the occurrence of an empty convex hexagon. 


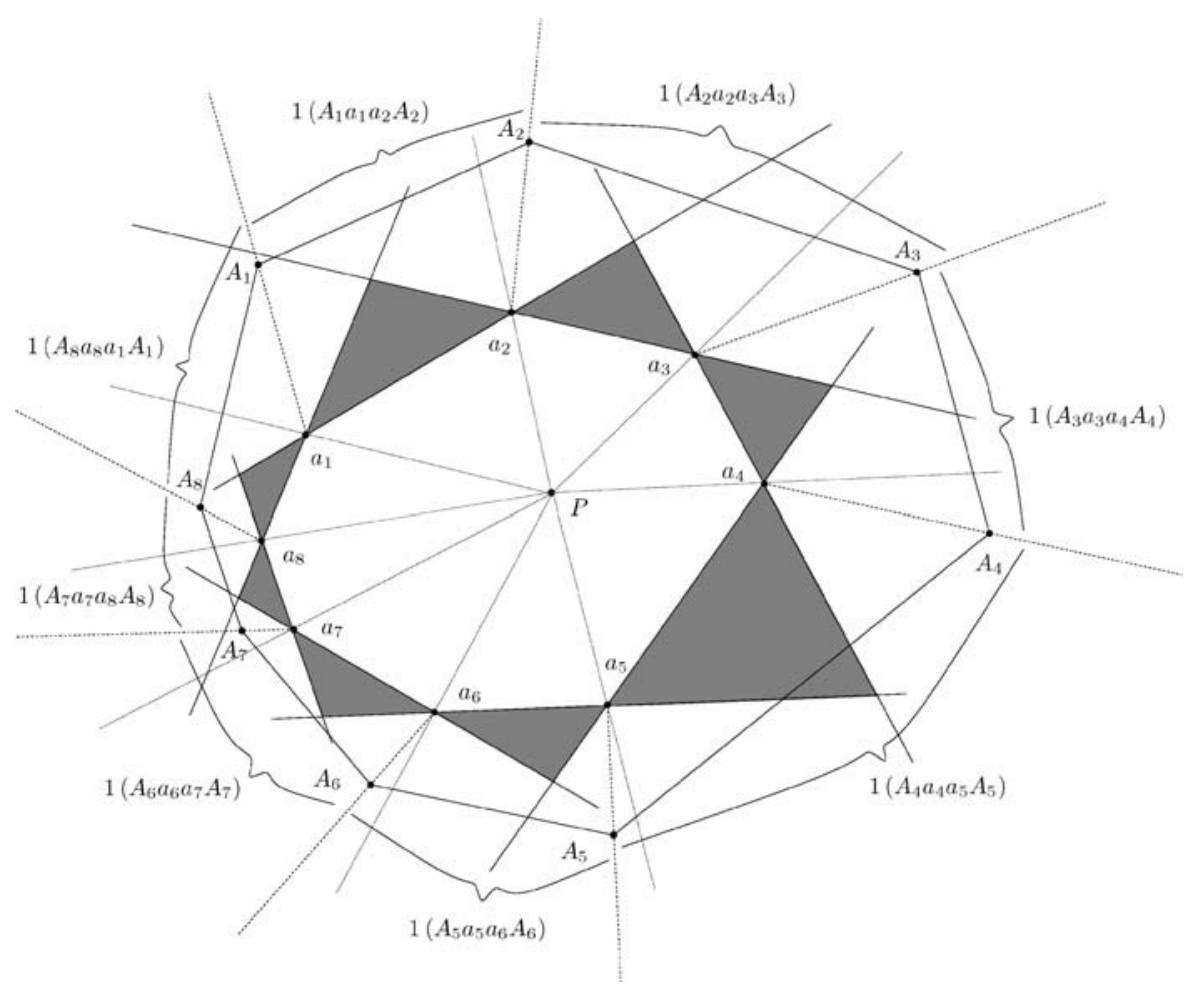

Fig. 35 The case $(8,8, \geq 1)$ with $(1,1,1,1,1,1,1,1): A_{1} \in R_{1} \backslash R_{2}$

Acknowledgements The author would like to thank Pavel Valtr and David Wood as well as the anonymous referees for their detailed comments on preliminary versions of this paper.

\section{References}

1. Bárány, I., Károlyi, Gy.: Problems and results around the Erdős-Szekeres convex polygon theorem. In: Discrete and Computational Geometry, Tokyo, 2000. Lect. Notes Comput. Sci., vol. 2098. Springer, Berlin (2001)

2. Brass, P., Moser, W., Pach, J.: Research Problems in Discrete Geometry. Springer, New York (2005)

3. Erdős, P.: Some more problems on elementary geometry. Aust. Math. Soc. Gaz. 5, 52-54 (1978)

4. Erdős, P.: Some applications of graph theory and combinatorial methods to number theory and geometry. In: Algebraic Methods in Graph Theory, vols. I, II, Szeged, 1978. Colloq. Math. Soc. János Bolyai, vol. 25, pp. 137-148. North-Holland, Amsterdam (1981)

5. Erdős, P., Szekeres, G.: A combinatorial problem in geometry. Compos. Math. 2, 463-470 (1935)

6. Erdős, P., Szekeres, G.: On some extremum problems in elementary geometry. Ann. Univ. Sci. Bp. Eötvös Sect. Math. 3-4, 53-62 (1960/1961)

7. Harborth, H.: Konvexe Fünfecke in ebenen Punktmengen. Elem. Math. 33, 116-118 (1978)

8. Horton, J.D.: Sets with no empty convex 7-gons. Can. Math. Bull. 26, 482-484 (1983)

9. Morris, W., Soltan, V.: The Erdôs-Szekeres problem on points in convex position-a survey. Bull. Am. Math. Soc. 37, 437-458 (2000)

10. Overmars, M.: Finding sets of points without empty convex 6-gons. Discrete Comput. Geom. 29, 153-158 (2003)

11. Tóth, G., Valtr, P.: The Erdős-Szekeres theorem: upper bounds and related results. In: Goodman, J.E., Pach, J., Welzl, E. (eds.) Combinatorial and Computational Geometry. MSRI Publications, vol. 52, pp. 557-568. Cambridge University Press, Cambridge (2005) 